This document is published in:

Chemical Engineering Journal, (2012), 198-199, 261-274.

DOI: http://dx.doi.org/10.1016/j.cej.2012.05.098

(C) 2012 Elsevier B.V. All rights reserved 


\title{
A novel methodology for simulating vibrated fluidized beds using two-fluid models
}

\author{
A. Acosta-Iborra*, F. Hernández-Jiménez, M. de Vega, J. V. Briongos \\ Department of Thermal and Fluid Engineering, Universidad Carlos III de Madrid, Avda. de la Universidad 30, 28911 Leganés, Madrid, Spain
}

\section{H I G H L I G H T S}

A novel strategy for the Eulerian simulation of vibrated fluidized beds is proposed.

- Vibration is incorporated through body forces in the transformed governing equations.

- Results compare well with DEM simulations and experiments for vertically vibrated beds.

- Phenomena like the bubble growth with the vibration strength are simulated.

\section{G R A P H I C A L A B S T R A C T}

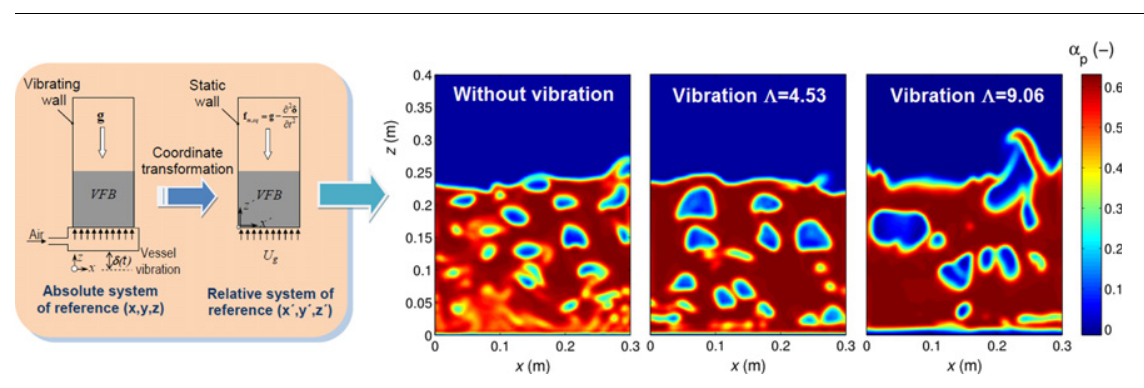

Keywords:

Fluidization

Two-fluid model

Vibrated fluidized bed

Vibration strength

Bubble

Body force

\begin{abstract}
A B S T R A C T
The present work considers the use of the two fluid (Euler Euler) CFD approach for the continuum description of vibrated fluidized beds as a less computationally demanding alternative to the discrete description given by Lagrangian Eulerian methods such as DEM. In particular, a novel simulation strat egy consisting on solving the two fluid model equations in a coordinate reference system that moves with the vibrating walls of a gas solid fluidized bed is proposed. By this way, vibration is transformed into simple alternating acceleration terms that are introduced through body forces in both the gas and the particle phase equations. The results of a series of two fluid model simulations compare well with discrete particle simulations as well as with experimental data reported for beds containing Geldart group B particles. In general, the results of a series of two fluid model simulations show similar trends to those seen in discrete particle simulations as well as in experimental data reported for beds containing Geldart group B particles. Exception of that is the velocity of bubbles, for which the two fluid simulations compare less satisfactorily with the available experimental data. The two fluid model simulations are also able to reproduce expected phenomena like the bubble growth with the vibration amplitude and the dependence of the pressure drop fluctuation on the vibration strength. In view of these promising results, the proposed two fluid model formulation opens the possibility of increasing the scale of the vibrated fluidized beds currently simulated.
\end{abstract}

\section{Introduction}

Fluidization is a process for powder handling widely used in industry owing to the favorable gas solid and solid solid contact ing efficiencies it provides. Nevertheless, gas fluidization alone has a limited ability to reduce particle agglomeration and gas channel ing. The vibrated fluidized bed (VFB) is a gas solid fluidization

\footnotetext{
* Corresponding author. Tel.: +34 916248465; fax: +34 916249430

E-mail address: aacosta@ing.uc3m.es (A. Acosta-Iborra).
}

technology that introduces an external vibration force to the con ventional fluidized bed (FB). Vibration prevents the solids from channeling and seriously agglomerating by supplying the bed with the energy required to break the bubble preferential paths and overcome the interparticle forces. At present, mechanical vibration has already been used in the processing of relatively large $(>1 \mathrm{~mm})$ as well as fine micron scale particles [ 16$]$ and very small nano particles in the range $1100 \mathrm{~nm}[7,8]$, and it has been proved to be an effective means in the fluidization of cohesive particles $[3,4,9]$, drying of granular material [10] and agglomeration control [8]. 


\section{Nomenclature}

A vertical vibration amplitude, $\mathrm{m}$

$d_{p} \quad$ particle diameter, $m$

$D_{b} \quad$ bubble equivalent diameter, $\mathrm{m}$

$e^{\text {restitution coefficient, dimensionless }}$

$f_{b} \quad$ bubble induced frequency in particle displacement, $\mathrm{Hz}$

$f \quad$ vertical vibration frequency, $\mathrm{Hz}$

$\mathbf{f}_{m, e q} \quad$ equivalent mass force vector, $\mathrm{m} / \mathrm{s}^{2}$

g gravity acceleration vector, $\mathrm{m} / \mathrm{s}^{2}$

$h_{0} \quad$ static bed height, $m$

$\mathrm{H} \quad$ total height of the bed vessel, $\mathrm{m}$

$\overline{\bar{I}} \quad$ unit tensor, dimensionless

$k_{\Theta} \quad$ granular temperature diffusion coefficient, $\mathrm{kg} / \mathrm{m} \mathrm{s}$

$K_{g p} \quad$ gas particle momentum exchange coefficient, $\mathrm{kg} / \mathrm{m}^{3} \mathrm{~s}$

$N_{i} \quad$ number of iterations per time step

$p \quad$ static pressure, $\mathrm{Pa}$

$t \quad$ time, $s$

$U_{g} \quad$ superficial gas velocity, $\mathrm{m} / \mathrm{s}$

$U_{m f} \quad$ minimum fluidization velocity, $\mathrm{m} / \mathrm{s}$

v velocity vector, $\mathrm{m} / \mathrm{s}$

W width of the bed, $\mathrm{m}$

$x \quad$ horizontal coordinate, $\mathrm{m}$

$z \quad$ vertical distance to the distributor, $\mathrm{m}$

\section{Greek letters}

$\alpha \quad$ volume fraction

$\boldsymbol{\delta}(t) \quad$ vibration displacement vector, $\mathrm{m}$
$\Delta t \quad$ time step, $\mathrm{s}$

$\Delta s \quad$ cell size, $\mathrm{m}$

$\phi \quad$ specularity coefficient, dimensionless

$\gamma_{\Theta} \quad$ collisional dissipation energy, $\mathrm{kg} / \mathrm{m} \mathrm{s}^{3}$

$\lambda$ bulk viscosity, Pa s

$\Lambda \quad$ vibration strength, dimensionless

$\mu \quad$ shear viscosity, Pa s

$\theta \quad$ friction angle, ${ }^{\circ}$

$\Theta \quad$ granular temperature, $\mathrm{m}^{2} / \mathrm{s}^{2}$

$\rho$ density, $\mathrm{kg} / \mathrm{m}^{3}$

$\bar{\tau} \quad$ shear stress tensor, Pa

$\nabla \quad$ gradient operator, $\mathrm{m}^{1}$

\section{Subscripts}

col collisional

fr frictional

$g \quad$ gas phase

$k \quad$ phase index

kin kinematic

max maximum

$p \quad$ particle phase

th threshold

w wall

$z \quad$ vertical direction
Experimental studies on the overall aerodynamics of vibrated fluidized beds have shown that VFB operates with lower minimum fluidization velocity, and pressure drop $[11,12]$ providing a higher homogeneity and stability of the fluidized bed. Bubble characteris tics such as bubble size, shape or ascending velocity are important parameters that have been extensively studied in conventional FB operating in the bubbling regime since they affect their perfor mance as reactors [13 17]. However, the literature on the effect of vibration on the bubble behavior is scarcer. Experiments by Zhou et al. $[18,19]$ and Mawatari et al. [20] visualized the differences in the bubble flow pattern without and with vibration in a two dimensional (2 D) VFB filled with glass beads of Geldart group B behavior [18,19] and $60 \mu \mathrm{m}$ particles [20]. The bubble sizes and local bubble rise velocities, at various gas velocities were measured while the bed was vibrated at different frequencies and amplitudes.

The dynamics of particle and gas phases in vibrated fluidized beds has been traditionally simulated employing the Lagrangian Eulerian approach (or discrete element method, DEM) that calcu lates the individual trajectory of each particle in the bed together with the solution of the continuum gas flow field. This allows the description of all the resulting particle particle and particle wall collisions [21,22]. Tatemoto et al. [23] and Xiang et al. [24] used the Lagrangian Eulerian method to simulate the motion of Geldart group B particles in a vibrated two dimensional bed that was fluidized with air. The thickness of the simulated bed was equal to the particle diameter and 1500 particles were considered. Changes in void fraction and air pressure fluctuations with time obtained from the simulation were analyzed and the distribution of particles in the bed was compared to experimental results [23]. Xiang et al. [24] also studied the distribution of velocity and concentration of particles at different amplitudes and frequencies of vibration, providing valuable qualitative information on the VFB behavior. Nevertheless, as stated in [24], quantitative evalua tion of the data was difficult since the number of particles in the calculation was too small. The Lagrangian Eulerian approach has been also employed in the simulation of three dimensional (3 D) VFB. A 3 D simulation of a cylindrical $(2.41 \mathrm{~mm}$ in diameter) vi brated fluidized bed filled with 14,135 cohesive fine particles was presented by Limtrakul et al. [25], where the effects of particle type, amplitude and frequency of vertical vibration, as well as the superficial gas velocity on the particle movement in the cylindrical bed were analyzed.

According to the literature reviewed, the use of discrete models for simulating VFB is restricted to beds of reduced dimensions, i.e. reduced number of particles, with the additional drawback of being highly computationally intensive [25]. Thus, a different computa tional strategy would be desirable to numerically simulate larger beds while achieving computing times similar to the ones required for the simulation of conventional FB. In this regard, simulation of small and medium scale conventional gas fluidized beds is com monly undertaken by means of two fluid computational fluid dynamic (CFD) models, also known as Eulerian Eulerian two fluid models, which are primarily based on the representation of the gas phase and the particulate phase as two interpenetrating con tinua $[22,26]$. Two fluid models provide information about the macroscopic hydrodynamics (i.e. velocity and volume fraction) of the two phases, including the bubble formation and motion in two and three dimensions [ [27 29].

The present work considers the use of the continuum descrip tion of VFB as a less computationally demanding alternative to the discrete description. In particular, a novel simulation strategy consisting on solving the two fluid model CFD equations in a coor dinate reference system that moves with the vibrating walls of the bed is proposed here. By this way vibration is transformed into simple alternating acceleration terms that are introduced through body forces in both the gas and the solids phases. To assess the capabilities of two fluid models in describing realistically vibrating fluidized beds, the results of a series of two fluid model simula tions are analyzed and compared with previously reported Lagrangian Eulerian simulations and experiments of sinusoidally 
Table 1

Governing equations in absolute coordinates.

\begin{tabular}{|c|c|c|}
\hline $\begin{array}{l}\text { Conservation of mass } \\
\frac{\partial}{\partial t}\left(\alpha_{g} \rho_{g}\right)+\nabla \cdot\left(\alpha_{g} \rho_{g} \mathbf{v}_{g}\right) \quad 0\end{array}$ & & (T1.1) \\
\hline$\frac{\partial}{\partial t}\left(\alpha_{p} \rho_{p}\right)+\nabla \cdot\left(\alpha_{p} \rho_{p} \mathbf{v}_{p}\right) \quad 0$ & & (T1.2) \\
\hline $\begin{array}{l}\text { Conservation of momentum } \\
\frac{\partial}{\partial t}\left(\alpha_{g} \rho_{g} \mathbf{v}_{g}\right)+\nabla \cdot\left(\alpha_{g} \rho_{g} \mathbf{v}_{g} \mathbf{v}_{g}\right)\end{array}$ & $\alpha_{g} \nabla p+\nabla \cdot\left(\alpha_{g} \tau_{g}\right) \quad K_{g p}\left(\mathbf{v}_{g} \quad \mathbf{v}_{p}\right)+\alpha_{g} \rho_{g} \mathbf{g}$ & (T1.3) \\
\hline$\frac{\partial}{\partial t}\left(\alpha_{p} \rho_{p} \mathbf{v}_{p}\right)+\nabla \cdot\left(\alpha_{p} \rho_{p} \mathbf{v}_{p} \mathbf{v}_{p}\right)$ & $\alpha_{p} \nabla p+\nabla p_{p}+\nabla \cdot\left(\alpha_{p} \tau_{p}\right)+K_{g p}\left(\mathbf{v}_{g} \quad \mathbf{v}_{p}\right)+\alpha_{p} \rho_{p} \mathbf{g}$ & (T1.4) \\
\hline $\begin{array}{l}\text { Balance of granular temperature } \\
\frac{3}{2}\left[\frac{\partial}{\partial t}\left(\rho_{p} \alpha_{p} \Theta\right)+\nabla \cdot\left(\rho_{p} \alpha_{p} \Theta \mathbf{v}_{p}\right)\right]\end{array}$ & $\left(p_{p} \overline{\bar{I}}+\tau_{p}\right): \nabla \mathbf{v}_{p}+\nabla \cdot\left(k_{\Theta} \nabla \Theta\right) \quad \gamma_{\Theta} \quad 3 K_{g p} \Theta$ & (T1.5) \\
\hline
\end{tabular}

vibrated beds filled with Geldart group B particles and fluidized with air in bubble regime.

\section{Theory}

\subsection{Two fluid model equations}

The description of gas fluidized beds as two interpenetrating continua is the essence of two fluid models $[22,26]$. One contin uum refers to the gas phase in the bed and the other to the particle phase (also known as particulate or solids phase). This allows the Eulerian description of both phases without resorting to the indi vidual tracking of each particle in the bed. Two fluid models make use of the general equations of conservation of mass and momen tum for the mass in the gas $(g)$ and particle $(p)$ phases. Table 1 lists these equations expressed in absolute coordinates and standard vector notation [22]. From the definition of volume fraction it is implicit that $\alpha_{g}+\alpha_{p}=1$. In the momentum equation, the particle phase is treated as a fluid with effective transport properties [26]. Commonly, the conservation equations of mass and momen tum are solved together with the differential equation for the transport of granular temperature $\Theta$, also included in Table 1 , which is based on the kinetic theory of granular flows and provides the level of random fluctuation of particle velocity due to collisions [26]. The granular temperature is required for the closure expres sions of the drag coefficient $K_{g p}$, the solid viscosities $\mu_{p}$ and $\lambda_{p}$, and the effective particle pressure $p_{p}$. Also functions of $\Theta$ are the diffusion coefficient of granular temperature $k_{\Theta}$ and the collision dissipation energy $\gamma_{\Theta}$. In the present work, the closure equation of Gidaspow et al. [30] has been chosen for calculating the drag coefficient $K_{g p}$ owing to its robustness at the beginning of the sim ulation sequence. Previous studies indicate that differences be tween this and other well known closure equations are not large in medium size two dimensional beds [28]. Table 2 summarizes these and other closure models selected for the present study.

\subsection{Transformed two fluid model equations}

As commented in Section 1, two fluid models have been tradi tionally employed for simulating non vibrating beds. However, as these models are based on universal laws of physics such as the mass conservation and the Newton's second law, nothing pre cludes their use in situations in which the walls vibrate. This is true provided the vibration has a frequency sufficiently smaller than the characteristic collision frequency of particles in the bed, that is, there is separation of scales between collision frequencies and wall vibration frequencies. At this regard, characteristic collisions times smaller than $10^{4} \mathrm{~s}$ (i.e. frequencies greater to $10^{4} \mathrm{~Hz}$ ) have been reported for conventional FB of Geldart B particles beyond the on set of bubbling [33]. In fact, bubble rise in conventional FB pushes upwards and downwards the particles within a region close to each bubble, the size of this region being of order of the bubble diameter [34]. The fluctuation induced by bubbles on the particle velocity has a characteristic frequency that can be roughly esti mated dividing the bubble velocity by the size of the perturbed area $f_{b} \approx 0.7 \sqrt{ } g D_{b} / D_{b} \approx 2 / \sqrt{ } D_{b}$. Hence, for bubble diameters of or der $110 \mathrm{~cm}$, the fluctuation induced by bubbles on the particle velocity in conventional FB has a characteristic frequency of order $10 \mathrm{~Hz}$, which is the same order of the vibration frequency consid ered in the present work. Therefore, for the vibrated bed regime studied here, it is expected the range of applicability of two fluid model equations to include vibration without the need of modify ing or adding new closure models.

In order to simulate vibration using two fluid Eulerian models, a direct imposition of vibration by means of oscillatory displace ments of the boundary condition at the walls of the bed has disad vantages related to the practical implementation of moving boundaries and the consistency of the mass and momentum bal ances in the computational cells at inlet flow boundaries (i.e. the bed distributor). In the present work, a novel strategy is presented to get rid of the disadvantages of the direct imposition of wall vibration. In particular, instead of using the traditional absolute system of reference for the spatial coordinates $\mathbf{x}=(x, y, z)$ in the governing equations, Eqs. (T1.1) (T1.5) in Table 1, a new relative system of reference $\mathbf{x}^{\prime}=\left(x^{\prime}, y^{\prime}, z^{\prime}\right)$ that moves attached to the bed vessel (i.e. bed walls) can be employed. Note that it is assumed that the walls are non deformable so that they move as a rigid solid. In this moving system of reference $\mathbf{x}^{\prime}$ there is no apparent movement of the walls and vibration information is incorporated in the gov erning equations through coordinate transformation factors. Thus, the computational mesh to be used for the solution of the govern ing equations is static in a moving system of reference, which greatly simplifies the numerical solution of the equations. Concep tually, the results should be equivalent to those obtained from the solution of the governing equations in the absolute coordinate sys tem using a mesh that moves with the bed walls. Fig. 1 sketches the procedure of coordinate transformation to a relative system of reference which moves with the bed vessel.

Without loss of generality, the movement of the bed walls due to vibration in an absolute system of reference will be expressed here as a sinusoidal displacement in any of the three spatial direc tions, but other displacement functions can be used: 
Table 2

Summary of closure models.

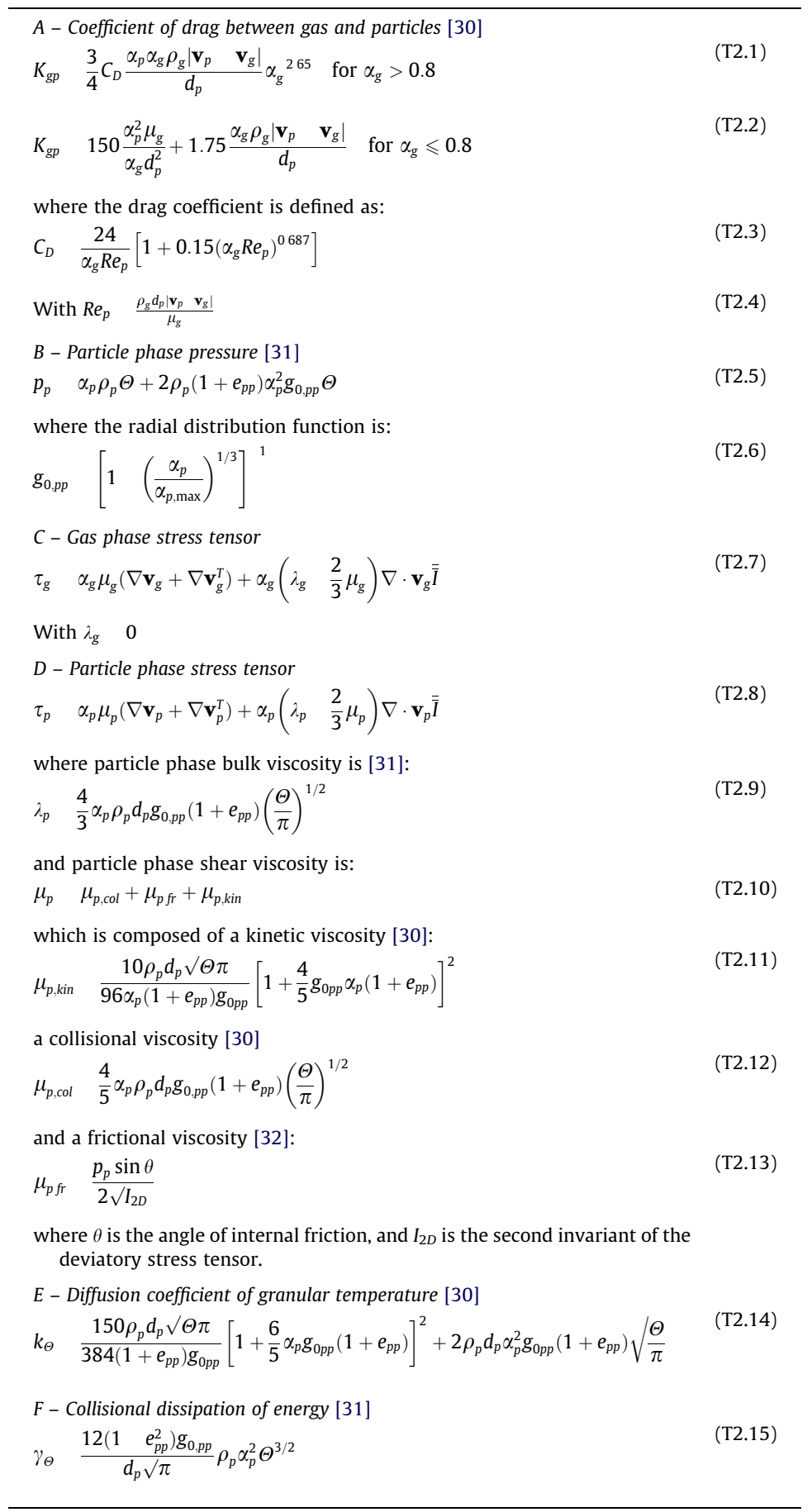

$\boldsymbol{\delta}(t) \quad\left\{a_{x} \sin \left(2 \pi f_{x} t\right), a_{y} \sin \left(2 \pi f_{y} t\right), a_{z} \sin \left(2 \pi f_{z} t\right)\right\}$

where $t$ is the time, the trio $a_{x}, a_{y}$ and $a_{z}$ represents the vibration amplitude in the $x, y$ and $z$ direction, respectively, and $f_{x}, f_{y}$ and $f_{z}$ are their corresponding vibration frequencies. Since the moving system of reference is attached to the bed walls the following transformation of coordinates is going to be used:

$t^{\prime} \quad t$

$\begin{array}{lll}\mathbf{X}^{\prime} & \mathbf{X} & \boldsymbol{\delta}\end{array}$
Thus, the gas or particle phase velocities expressed in the mov ing system of reference are

$\mathbf{v}^{\prime} \quad \mathbf{v} \quad \partial \boldsymbol{\delta} / \delta t$

Applying the chain rule it is easy to demonstrate that

$\frac{\partial}{\partial t} \quad \frac{\partial}{\partial t^{\prime}} \quad \frac{\partial \boldsymbol{\delta}}{\partial t} \cdot \nabla^{\prime}$

$\nabla \quad \nabla^{\prime}$ 


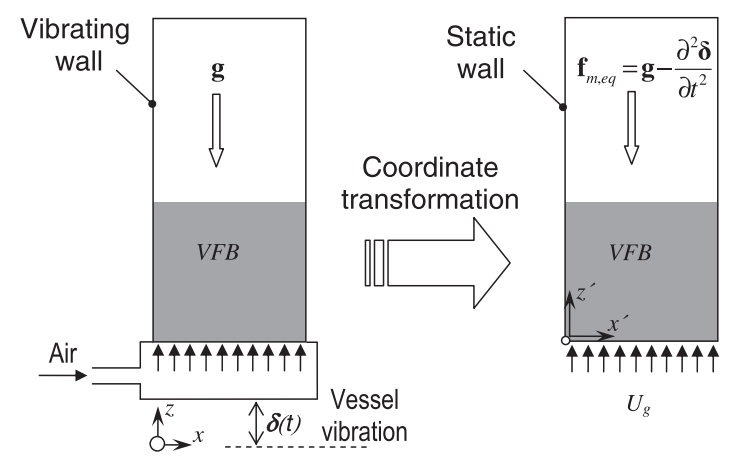

Absolute system of reference $(x, y, z)$

Relative system of reference $\left(x^{\prime}, y^{\prime}, z^{\prime}\right)$

Fig. 1. Transformation of coordinates from an absolute to a relative system of reference attached to the bed vessel in a vibrating fluidized bed (VFB).

where $\nabla^{\prime}=\left\{\partial / \partial x^{\prime}, \partial / \partial y^{\prime}, \partial / \partial z^{\prime}\right\}$ is the gradient operator in the mov ing system of reference. Note also that $\partial \boldsymbol{\delta} / \partial t=\partial \boldsymbol{\delta} / \partial t^{\prime}$. Introducing the transformed time derivative and gradient in Eq. (T1.1),

$\frac{\partial}{\partial t^{\prime}}\left(\alpha_{g} \rho_{g}\right) \quad \frac{\partial \boldsymbol{\delta}}{\partial t^{\prime}} \cdot \nabla^{\prime}\left(\alpha_{g} \rho_{g}\right)+\nabla^{\prime} \cdot\left(\alpha_{g} \rho_{g} \mathbf{v}_{g}\right) \quad 0$

and using Eq. (3) together with the fact that $\partial \delta / \delta t \quad \nabla^{\prime}\left(\alpha_{g} \rho_{g}\right)=$ $\nabla^{\prime} \quad\left(\alpha_{g} \rho_{g} \partial \delta / \delta t\right)$ since $\delta$ is only a function of $t$, the equation of mass conservation for the gas phase after transforming coordinates is shown in Eq. (T3.1) in Table 3, which is analogous to the equation in the absolute system of coordinates. This result should be expected since the conservation of mass is a property independent of the system of reference. A similar result is obtained for the conservation of mass in transformed coordinates for the particle phase, Eq. (T3.2) in Table 3.

The same transformation of coordinates can be done for the momentum equations:

$$
\begin{aligned}
& \frac{\partial}{\partial t^{\prime}}\left(\alpha_{g} \rho_{g} \mathbf{v}_{g}\right) \quad \frac{\partial \boldsymbol{\delta}}{\partial t^{\prime}} \cdot \nabla^{\prime}\left(\alpha_{g} \rho_{g} \mathbf{v}_{g}\right)+\nabla^{\prime} \cdot\left(\alpha_{g} \rho_{g} \mathbf{v}_{g} \mathbf{v}_{g}\right) \\
& \alpha_{g} \nabla^{\prime} p+\nabla^{\prime} \cdot\left(\alpha_{g} \tau_{g}\right) \quad K_{g p}\left(\mathbf{v}_{g} \quad \mathbf{v}_{p}\right)+\alpha_{g} \rho_{g} \mathbf{g} \\
& \frac{\partial}{\partial t^{\prime}}\left(\alpha_{p} \rho_{p} \mathbf{v}_{p}\right) \quad \frac{\partial \boldsymbol{\delta}}{\partial t^{\prime}} \cdot \nabla^{\prime}\left(\alpha_{p} \rho_{p} \mathbf{v}_{p}\right)+\nabla^{\prime} \cdot\left(\alpha_{p} \rho_{p} \mathbf{v}_{p} \mathbf{v}_{p}\right) \\
& \alpha_{p} \nabla^{\prime} p+\nabla^{\prime} p_{p}+\nabla^{\prime} \cdot\left(\alpha_{p} \tau_{p}\right)+K_{g p}\left(\mathbf{v}_{g} \quad \mathbf{v}_{p}\right)+\alpha_{p} \rho_{p} \mathbf{g}
\end{aligned}
$$

Again, incorporating Eq. (3) in Eqs. (6) and (7) and realizing that $\nabla^{\prime}(\partial \boldsymbol{\delta} / \partial t)=0$ and

$$
\mathbf{v v} \quad \mathbf{v}^{\prime} \mathbf{v}^{\prime}+\mathbf{v}^{\prime} \frac{\partial \boldsymbol{\delta}}{\partial t}+\frac{\partial \boldsymbol{\delta}}{\partial t} \mathbf{v}^{\prime}+\frac{\partial \boldsymbol{\delta}}{\partial t} \frac{\partial \boldsymbol{\delta}}{\partial t}
$$

the following transformed momentum equations are obtained:

$$
\begin{aligned}
& \frac{\partial}{\partial t^{\prime}}\left(\alpha_{g} \rho_{g} \mathbf{v}_{g}^{\prime}\right)+\frac{\partial}{\partial t^{\prime}}\left(\alpha_{g} \rho_{g} \frac{\partial \boldsymbol{\delta}}{\partial t^{\prime}}\right) \quad \frac{\partial \boldsymbol{\delta}}{\partial t^{\prime}} \cdot \nabla^{\prime}\left(\alpha_{g} \rho_{g} \mathbf{v}_{g}^{\prime}\right) \quad \frac{\partial \boldsymbol{\delta}}{\partial t^{\prime}} \\
& \nabla^{\prime}\left(\alpha_{g} \rho_{g} \frac{\partial \boldsymbol{\delta}}{\partial t^{\prime}}\right)+\nabla^{\prime} \cdot\left(\alpha_{g} \rho_{g} \mathbf{v}_{g}^{\prime} \mathbf{v}_{g}^{\prime}\right)+\nabla^{\prime} \cdot\left(\alpha_{g} \rho_{g} \mathbf{v}_{g}^{\prime} \frac{\partial \boldsymbol{\delta}}{\partial t^{\prime}}\right)+\nabla^{\prime} \\
& \left(\alpha_{g} \rho_{g} \frac{\partial \boldsymbol{\delta}}{\partial t^{\prime}} \mathbf{v}_{g}^{\prime}\right)+\nabla^{\prime} \cdot\left(\alpha_{g} \rho_{g} \frac{\partial \boldsymbol{\delta}}{\partial t^{\prime}} \frac{\partial \boldsymbol{\delta}}{\partial t^{\prime}}\right) \\
& \alpha_{g} \nabla^{\prime} p+\nabla^{\prime} \cdot\left(\alpha_{g} \tau_{g}^{\prime}\right) \quad K_{g p}\left(\mathbf{v}_{g}^{\prime} \quad \mathbf{v}_{p}^{\prime}\right)+\alpha_{g} \rho_{g} \mathbf{g} \\
& \frac{\partial}{\partial t^{\prime}}\left(\alpha_{p} \rho_{p} \mathbf{v}_{p}^{\prime}\right)+\frac{\partial}{\partial t^{\prime}}\left(\alpha_{p} \rho_{p} \frac{\partial \boldsymbol{\delta}}{\partial t^{\prime}}\right) \quad \frac{\partial \boldsymbol{\delta}}{\partial t^{\prime}} \cdot \nabla^{\prime}\left(\alpha_{p} \rho_{p} \mathbf{v}_{p}^{\prime}\right) \quad \frac{\partial \boldsymbol{\delta}}{\partial t^{\prime}} \\
& \nabla^{\prime}\left(\alpha_{p} \rho_{p} \frac{\partial \boldsymbol{\delta}}{\partial t^{\prime}}\right)+\nabla^{\prime} \cdot\left(\alpha_{p} \rho_{p} \mathbf{v}_{p}^{\prime} \mathbf{v}_{p}^{\prime}\right)+\nabla^{\prime} \cdot\left(\alpha_{p} \rho_{p} \mathbf{v}_{p}^{\prime} \frac{\partial \boldsymbol{\delta}}{\partial t^{\prime}}\right)+\nabla^{\prime} \\
& \left(\alpha_{p} \rho_{p} \frac{\partial \boldsymbol{\delta}}{\partial t^{\prime}} \mathbf{v}_{p}^{\prime}\right)+\nabla^{\prime} \cdot\left(\alpha_{p} \rho_{p} \frac{\partial \boldsymbol{\delta}}{\partial t^{\prime}} \frac{\partial \boldsymbol{\delta}}{\partial t^{\prime}}\right) \\
& \alpha_{p} \nabla^{\prime} p+\nabla^{\prime} p_{p}+\nabla^{\prime} \cdot\left(\alpha_{p} \tau_{p}^{\prime}\right)+K_{g p}\left(\mathbf{v}_{g}^{\prime} \quad \mathbf{v}_{p}^{\prime}\right)+\alpha_{p} \rho_{p} \mathbf{g}
\end{aligned}
$$

where $\overline{\bar{\tau}}_{k}^{\prime} \quad \mu_{k}\left(\nabla^{\prime} \mathbf{v}_{k}^{\prime}+\nabla^{\prime} \mathbf{v}_{k}^{\prime T}\right)+\left(\begin{array}{ll}\lambda_{k} & \frac{2}{3} \mu_{k}\end{array}\right)\left(\begin{array}{ll}\nabla^{\prime} & \mathbf{v}_{k}^{\prime}\end{array}\right) \overline{\bar{I}}$ is the tensor of shear stresses for the velocities of the gas $(k=g)$ and particle $(k=p)$ phases in the moving coordinates.

The transformed momentum conservation expressions, Eqs. (9) and (10), can be further simplified noticing that $\frac{\partial \delta}{\partial t^{\prime}} \nabla^{\prime}\left(\alpha_{g} \rho_{g} \frac{\partial \delta}{\partial t^{\prime}}\right)$

$\nabla^{\prime}\left(\alpha_{g} \rho_{g} \frac{\partial \delta}{\partial t^{\prime}} \frac{\partial \delta}{\partial t^{\prime}}\right) \quad$ and $\frac{\partial}{\partial t^{\prime}}\left(\alpha \rho \frac{\partial \delta}{\partial t^{\prime}}\right) \quad \frac{\partial \delta}{\partial t^{\prime}} \quad \nabla^{\prime}\left(\alpha \rho \mathbf{v}^{\prime}\right)+\nabla^{\prime}\left(\alpha \rho \mathbf{v}^{\prime} \frac{\partial \delta}{\partial t^{\prime}}\right)$ $+\nabla^{\prime} \quad\left(\alpha \rho \frac{\partial \delta}{\partial t^{\prime}} \mathbf{v}^{\prime}\right) \quad \alpha \rho \frac{\partial^{2} \delta}{\partial t^{\prime 2}}+\frac{\partial \delta}{\partial t^{\prime}}\left[\frac{\partial}{\partial t^{\prime}}(\alpha \rho)+\nabla^{\prime}\left(\alpha \rho \mathbf{v}^{\prime}\right)\right]$.

Besides, $\frac{\partial \delta}{\partial t^{\prime}}\left[\frac{\partial}{\partial t^{\prime}}(\alpha \rho)+\nabla^{\prime}\left(\alpha \rho \mathbf{v}^{\prime}\right)\right] \quad 0$ owing to the conservation of mass, Eqs. (T3.1) and (T3.2). This leads to the final form of the transformed equations for the momentum conservation included in Table 3, Eqs. (T3.3) and (T3.4).

Regarding the granular temperature $\Theta$, it accounts for the qua dratic difference between the individual velocity of particles and the bulk velocity $\mathbf{v}_{p}$ of the particle phase [26]. By definition this velocity difference is stochastic, which implies that changing from a static to a moving system of reference modifies the bulk velocity as well as the individual velocity of particles, but does not alter the difference between individual and bulk velocities. This can be seen

Table 3

Governing equations in moving coordinates: transformed two-fluid model equations.

$$
\begin{aligned}
& \text { Conservation of mass } \\
& \frac{\partial}{\partial t^{\prime}}\left(\alpha_{g} \rho_{g}\right)+\nabla^{\prime} \cdot\left(\alpha_{g} \rho_{g} \mathbf{v}_{g}^{\prime}\right) \quad 0 \\
& \frac{\partial}{\partial t^{\prime}}\left(\alpha_{p} \rho_{p}\right)+\nabla^{\prime} \cdot\left(\alpha_{p} \rho_{p} \mathbf{v}_{p}^{\prime}\right) \quad 0 \\
& \frac{\partial}{\partial t^{\prime}}\left(\alpha_{g} \rho_{g} \mathbf{v}_{g}^{\prime}\right)+\nabla^{\prime} \cdot\left(\alpha_{g} \rho_{g} \mathbf{v}_{g}^{\prime} \mathbf{v}_{g}^{\prime}\right) \quad \alpha_{g} \nabla^{\prime} p+\nabla^{\prime} \cdot\left(\alpha_{g} \tau_{g}^{\prime}\right) \quad K_{g p}\left(\begin{array}{ll}
\mathbf{v}_{g}^{\prime} & \mathbf{v}_{p}^{\prime}
\end{array}\right)+\alpha_{g} \rho_{g}\left(\begin{array}{ll}
\mathbf{g} & \frac{\partial^{2} \boldsymbol{\delta}}{\partial t^{\prime 2}}
\end{array}\right) \\
& \frac{\partial}{\partial t^{\prime}}\left(\alpha_{p} \rho_{p} \mathbf{v}_{p}^{\prime}\right)+\nabla^{\prime} \cdot\left(\alpha_{p} \rho_{p} \mathbf{v}_{p}^{\prime} \mathbf{v}_{p}^{\prime}\right) \quad \alpha_{p} \nabla^{\prime} p+\nabla^{\prime} p_{p}+\nabla^{\prime} \cdot\left(\alpha_{p} \tau_{p}^{\prime}\right)+K_{g p}\left(\mathbf{v}_{g}^{\prime} \quad \mathbf{v}_{p}^{\prime}\right)+\alpha_{p} \rho_{p}\left(\begin{array}{ll}
\mathbf{g} & \left.\frac{\partial^{2} \delta}{\partial t^{\prime 2}}\right)
\end{array}\right.
\end{aligned}
$$

Balance of granular temperature

$\frac{3}{2}\left[\frac{\partial}{\partial t^{\prime}}\left(\rho_{p} \alpha_{p} \Theta\right)+\nabla^{\prime} \cdot\left(\rho_{p} \alpha_{p} \Theta \mathbf{v}_{p}^{\prime}\right)\right] \quad\left(p_{p} \overline{\bar{I}}+\tau_{p}^{\prime}\right): \nabla^{\prime} \mathbf{v}_{p}^{\prime}+\nabla^{\prime} \cdot\left(k_{\Theta} \nabla^{\prime} \Theta\right) \quad \gamma_{\Theta} \quad 3 K_{g p} \Theta$ 
noticing that both the individual and bulk velocities of particles in a moving system of reference are modified by the same apparent velocity, $\partial \boldsymbol{\delta} / \partial t$. Thus, this apparent velocity vanishes when calcu lating the difference between the individual and bulk particle velocities. Therefore, the granular temperatures observed from the moving and the static systems of reference are equal, i.e. $\Theta^{\prime}=\Theta$, which means that, for a given bubbling bed, the observed granular temperature is independent of the system of reference. As for the case of the mass conservation equations, transformation of coordinates and velocities using Eqs. 3, 4.a, 4.b, 5 leave unchanged the structure of the governing equation for the granular temperature, Eq. (T3.5) in Table 3.

All the deductions above have been done for two phases (i.e. gas and particles), but the same procedure can be equally carried out in beds containing a mixture of gas, liquid and solid phases or multi ple particle phases representing differentiated particle size distributions.

\subsection{Acceleration and equivalent mass forces}

In the previous Section 2.2 the governing equations for the gas and particulate phases expressed in a coordinate system that moves with the walls of the vibrated bed have been deduced. As noticed previously, the resulting equations for the mass conserva tion and granular temperature balance, Eqs. (T3.1), (T3.2) and (T3.5), are similar to the equations in a static system of reference, Eqs. (T1.1), (T1.2) and (T1.5). In contrast, the equations of momen tum conservation in a moving system of reference, Eqs. (T3.3) and (T3.4), differ from the original ones, Eqs. (T1.3) and (T1.4), in that they posses an extra term in their right hand side related to the acceleration of the system of reference, $\partial^{2} \delta / \partial t^{\prime 2}$. In fact this accel eration term can be viewed as an apparent volumetric force, acting over both the gas and the particulate phases, that reflects the coun ter reaction to the acceleration of the system of reference. Therefore,

$\mathbf{f}_{m, e q} \quad \mathbf{g} \quad \frac{\partial^{2} \boldsymbol{\delta}}{\partial t^{\prime 2}}$

is the equivalent vector of mass force that should be implemented in the model, instead of the gravity vector $\mathbf{g}$, to simulate a vibrating bed with two fluid models. For a sinusoidal vibration, Eq. (1), the equivalent vector of mass force is

$$
\begin{aligned}
\mathbf{f}_{m, e q} & \left\{g_{x}+4 \pi^{2} f_{x}^{2} a_{x} \sin \left(2 \pi f_{x} t\right), g_{y}+4 \pi^{2} f_{y}^{2} a_{y} \sin \left(2 \pi f_{y} t\right), g_{z}\right. \\
& \left.+4 \pi^{2} f_{z}^{2} a_{z} \sin \left(2 \pi f_{z} t\right)\right\}
\end{aligned}
$$

Obviously, after solving the system of transformed equations, Eqs. (T3.1) (T3.5) in Table 3, the absolute position $\mathbf{x}$ of the phases, the velocity $\mathbf{v}$, and the shear stresses $\tau$, can be recuperated by undoing the transformation given by Eqs. (2) and (3).

\subsection{Effect of the transformation on the boundary conditions}

It is to be noticed that in the transformed coordinates, given by the moving system of reference, all the vibration effects in the model are concentrated in Eqs. (T3.3) and (T3.4), whereas the apparent movement of the walls and other boundaries is null. That means that the boundary conditions to be employed by the trans formed equations in Table 3, are similar to the standard conditions used in a non vibrating bed. Implicit in this reasoning is the assumption that the plenum and the distributor vibrate attached to the rest of the bed vessel. Thus, the gas superficial velocity in the moving system of reference is steady and only depends on the gas flow rate entering the plenum as in a non vibrating bed.

\section{Simulation and postprocessing}

\subsection{Bed configurations}

Two different bed configurations are studied in the present work in order to analyze the capabilities of the two fluid model in predicting realistically the dynamics of vibrating fluidized beds. The two configurations are filled with spherical particles of uni form size and Geldart B group behavior. For all the simulations the standard downward gravity acceleration will be considered, $\mathbf{g}=\{0,0,9.81\} \mathrm{m}^{2} / \mathrm{s}$.

\subsubsection{Configuration 1: slim bed}

The first configuration (configuration 1) consists of a slim bed that has been subject to previous Lagrangian Eulerian (DEM) sim ulations $[23,24]$. This configuration has been selected here to ana lyze the consistency of two fluid models with the DEM simulation strategy. The bed is a rectangular column of reduced dimensions containing 1500 particles. As the bed thickness coincides to one particle diameter, the gas and particle motion can be regarded two dimensional. The bed is fluidized with air and its vessel verti cally moved with sinusoidal vibration, Eq. (1). The air is uniformly injected, in time and space, from below into the bed at a superficial velocity $U_{g}$ that is nearly twice the minimum fluidization velocity $U_{m f}$ in the bed without vibration [23].

\subsubsection{Configuration 2: high amplitude vibrating bed}

The second configuration (configuration 2) is a two dimen sional bed of medium size. As in the first configuration, a steady flux of air is uniformly injected through the base of the bed and vibration of the bed vessel is sinusoidal and vertically oriented. The vessel vibration amplitude is relatively high (of order $10 \mathrm{~mm}$ ). This bed configuration is similar to the two dimensional bed of $10 \mathrm{~mm}$ thickness used by Zhou et al. [19] in their experi mental work, and has been chosen here to analyze the consistency of the two fluid simulation results with experiments. To carry out the numerical simulations of the bed in the present work, the air superficial velocity was set to $U_{g} \approx 1.3 U_{m f}$.

Information comprising the bed geometry and operative condi tions, together with the air and particle properties and other sim ulation parameters, is included in Table 4 for the two vibrated beds configurations.

\subsection{Numerical solution}

\subsubsection{Simulation domain and mesh}

The two bed configurations described in the previous section were simulated in two dimensions using meshes composed of quadrilateral cells spanning the entire bed domain. The height of the bed walls, which was reduced to $H=0.25 \mathrm{~m}$ for the slim bed (configuration 1) to lower the computational cost. In contrast, the height of the bed walls was doubled $(H=1.2 \mathrm{~m})$ in the second bed configuration (configuration 2 ) to ensure the stability of the simulation at the top of the freeboard for large vibration ampli tudes (e.g. $A=15 \mathrm{~mm}$ ). Figs. 2a and 3a illustrate the dimensions of the simulated domain together with the mesh used for the two bed configurations. A snapshot of particle volume fraction ta ken from the two fluid simulations is included in Fig. $2 b$ and in Fig. $3 \mathrm{~b}$ to show the size of the fluidized bed relative to the domain dimensions.

Tables 4 and 5 list all the simulated cases. In all the computa tional meshes used in the cases the size of the cells is reduced in the region occupied by the bed particles. Above the bed surface, i.e. the freeboard, the size of the computational cells was gradually increased to diminish the total number of cells. Case SB 2 was se 
Table 4

Summary of simulation parameters.

\begin{tabular}{lll}
\hline Parameter & $\begin{array}{l}\text { Configuration-1: vibrating } \\
\text { slim bed }\end{array}$ & $\begin{array}{l}\text { Configuration-2: high-amplitude } \\
\text { vibrating bed }\end{array}$ \\
\hline$W(\mathrm{~mm})$ & 25 & 300 \\
$H(\mathrm{~mm})$ & 250 & 600 \\
$h_{0}(\mathrm{~mm})$ & 55 & 205 \\
$d_{p}(\mu \mathrm{m})$ & 1000 & 198 \\
$\rho_{p}\left(\mathrm{~kg} / \mathrm{m}^{3}\right)$ & 1050 & 2520 \\
$\rho_{g}\left(\mathrm{~kg} / \mathrm{m}^{3}\right)$ & 1.167 & 1.167 \\
$\mu_{\mathrm{g}}(\mathrm{Pa} \mathrm{s})$ & $1.82 \mathrm{e}-5$ & $1.82 \mathrm{e}-5$ \\
$U_{g}(\mathrm{~m} / \mathrm{s})$ & 0.5 & 0.0907 \\
$f(\mathrm{~Hz})$ & $5-30$ & $15-30$ \\
$A(\mathrm{~mm})$ & $1-2$ & $5-15$ \\
$e_{p p}(-)$ & 0.9 & 0.9 \\
$e_{p w}(-)$ & 0.9 & 0.2 \\
$\alpha_{p f r}(-)$ & 0.6 & 0.61 \\
$\alpha_{p, \max }(-)$ & 0.605 & 0.63 \\
$\theta\left({ }^{\circ}\right)$ & 30 & 30 \\
$\phi(-)$ & 0.6 & 0.6 \\
$\Delta t(\mathrm{~s})$ & $2.5 \mathrm{e}-4$ & $1 \mathrm{e}-4$ \\
$N_{i}($ iterations $)$ & 40 & 50 \\
\hline
\end{tabular}

lected as the base case of the slim bed, with vibration frequency equal to $15 \mathrm{~Hz}$ and amplitude $1.5 \mathrm{~mm}$. Similarly, case HA 2 (with vibration frequency $15 \mathrm{~Hz}$ and amplitude $15 \mathrm{~mm}$ ) was utilized as the base case for the simulation of the high amplitude vibrated bed. For these base cases, as well as for the non vibrated cases, there are three different meshes used to analyze the simulation sensitivity on the mesh number of nodes.

\subsubsection{Boundary condition parameters}

A time independent and spatially uniform superficial velocity condition, $U_{g}$ in Table 4, was imposed at the bottom of both bed configurations for the gas phase, see Figs. 2b and 3b. Null velocity and concentration were set for the particle phase in order to avoid it to cross the distributor. Pressure outlet conditions (denoted as PO in Figs. 2b and $3 \mathrm{~b}$ ) were selected for the upper boundary of

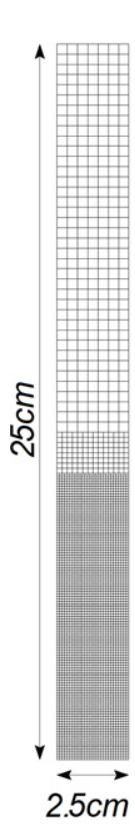

(a)

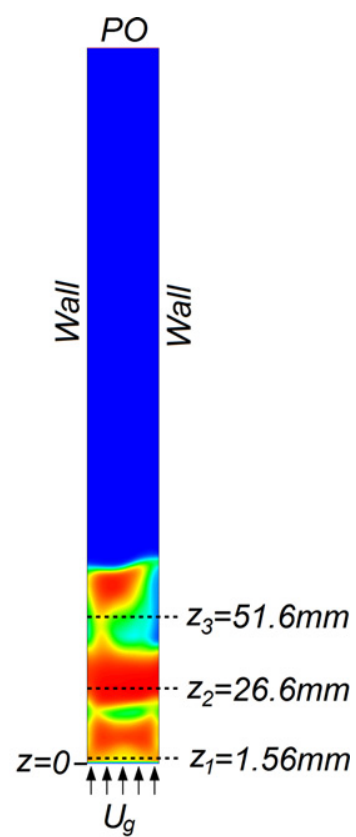

(b)
Fig. 2. Configuration-1 consisting in a vibrated slim bed: (a) domain dimensions and computational mesh used for the two-fluid simulation; (b) boundary conditions in relative coordinates and vertical positions for data sampling.

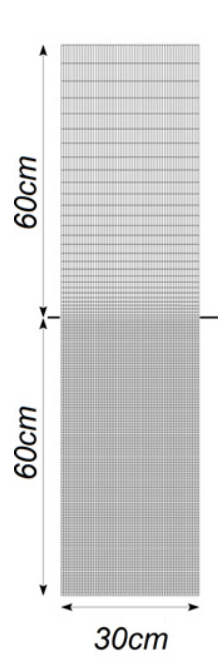

(a)

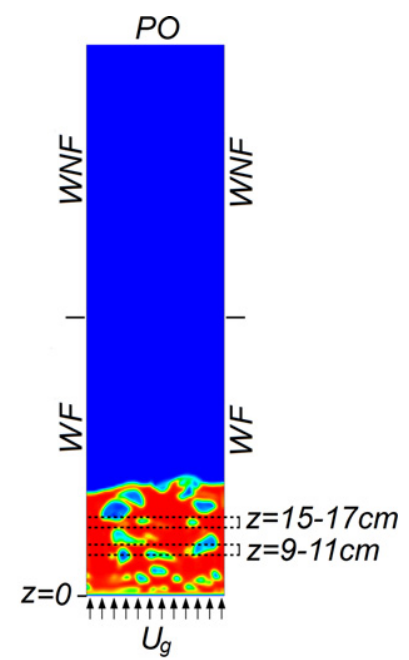

(b)
Fig. 3. Configuration- 2 consisting in a fluidized bed with high-amplitude vibration: (a) domain dimensions and computational mesh used for the two-fluid simulation; (b) boundary conditions in relative coordinates and data sampling regions.

the domain with static pressure equal to one atmosphere. At the lateral walls, considered static in the moving coordinates as ex plained in Section 2.4, non slip conditions were assigned to the gas phase, and partial slip conditions were assumed for the parti cle phase velocity using a standard specularity coefficient, $\phi=0.6$ [35]. Besides, Johnson and Jackson's boundary conditions [35] were employed for the granular temperature at the lateral walls of the bed. For the slim bed (configuration 1), in accordance to the DEM simulations by Tatemoto et al. [23] and Xiang et al. [24], the parti cle to wall restitution coefficient was set to $e_{p w}=0.9$. For the high amplitude vibrating bed (configuration 2) the wall restitution coefficient was lowered to the code default value $\left(e_{p w}=0.2\right)$ since no experimental information at this regard was available. In the configuration 2 cases, in order to confine the effects of wall friction to an effective height of $600 \mathrm{~mm}$ similar to that of the experiment of Zhou et al. [19], wall friction on the upper half to the bed walls (i.e. from $z=0.61 .2 \mathrm{~m}$ ) was eliminated from the boundary condi tions of the simulation. This is indicated in Fig. 3b, where the walls with friction are labeled with WF while the walls having no friction are marked with WNF.

\subsubsection{Solution procedure}

The commercial code ANSYS FLUENT v12.0 was used to solve the transformed two fluid model differential equations of continu ity and momentum, Eqs. (T3.1) (T3.4) for the gas and particle phases together with the granular temperature balance equation, Eq. (T3.5). This code resorts to a finite volume formulation with an implicit time advance algorithm to solve these transient govern ing equations, the coupling between the gas and particle phases being done by a modified SIMPLE algorithm (Phase coupled SIM PLE), which has given satisfactory results in the prediction of the pressure and bubble behavior of conventional fluidized beds [17]. Since the transformed governing equations to be solved are ex pressed in a moving system of coordinates, vibration of the bed vessel was incorporated in the simulation through the equivalent vector of mass force, Eq. (12), which was programmed in a sepa rated $\mathrm{C}++$ code and interpreted by ANSYS FLUENT as a user defined function. A second order accuracy scheme was selected for the discretization of the advective transport terms as well as for the time advance algorithm.

The restitution coefficient for the collisions between particles was $e_{p p}=0.9$ for all the simulations. For the slim bed (configura 
Table 5

Two-fluid simulation cases for the slim bed (configuration-1).

\begin{tabular}{llll}
\hline Cases & $\begin{array}{l}\text { Mesh nodes (nodes in } \\
\text { horizontal direction) }\end{array}$ & $\begin{array}{l}\text { Vibration } \\
\text { frequency } f(\mathrm{~Hz})\end{array}$ & $\begin{array}{l}\text { Vibration } \\
\text { amplitude } A(\mathrm{~mm})\end{array}$ \\
\hline SB-1A & $1279(14)$ & No vibration & - \\
SB-1B & $3701(28)$ & & \\
SB-1C & $12,314(56)$ & & \\
SB-2A & $1279(14)$ & 15 & 1.5 \\
SB-2B & $3701(28)$ & 15 & 1.5 \\
SB-2C & $12,314(56)$ & 15 & 1.5 \\
SB-3 & $3701(28)$ & 5 & 1.5 \\
SB-4 & $3701(28)$ & 20 & 1.5 \\
SB-5 & $3701(28)$ & 30 & 1.5 \\
SB-6 & $3701(28)$ & 15 & 1 \\
SB-7 & $3701(28)$ & 15 & 2 \\
\hline
\end{tabular}

tion 1) the maximum packet limit was assumed to be $\alpha_{p \text { - }}$ $\max =0.605$, which is calculated from a hexagonal arrangement of spheres occupying a bed of thickness equal to their diameter. For the high amplitude vibrating bed (configuration 2), since the bed thickness is several times the particle diameter, the standard value $\alpha_{p \text { max }}=0.63$ was chosen to represent the maximum packing limit. In all the simulations the value chosen for the friction packing limit $\alpha_{p . f r}$ was slightly smaller than $\alpha_{p \text {.max }}$. It was found that differences between these two packing limits did not affect markedly the sim ulation results, though convergence of the solution seemed to pro ceed smoothly if $\alpha_{p . f r}$ was inferior to $\alpha_{p \text { max. }}$.

The simulations commenced with air entering at the distributor with the bed at rest. The time step of the simulations was fixed to values of order $10^{4} \mathrm{~s}$, that is, two orders of magnitude smaller than the characteristic vibration time period. The number of itera tions per time step, $N_{i}$, was 40 and 50 (see Table 4 ). This ensured that the residuals of the numerically solved equations remained below $10^{3}$. To obtain statistically steady state results, a total sim ulated time of more than $25 \mathrm{~s}$ for the slim bed configuration and $12 \mathrm{~s}$ for the high amplitude bed configuration were computed. The first $4 \mathrm{~s}$ of simulations were not used in the results in order to eliminate the transient effects during the fluidization start up.

\section{Results}

\subsection{Mesh sensitivity analysis}

Three different meshes were studied for each vibrating bed con figuration in order to assess the sensitivity of the simulation on the spatial resolution of the computational mesh. The mesh sensitivity analysis was focused on the non vibrating case as well as the base cases for the vibrating beds. For the slim bed (configuration 1), cases SB $1 \mathrm{~A}$ and SB 2A in Table 5 were solved on a mesh having cells of size $\Delta s=1.78 \mathrm{~mm}$ in both $x$ (horizontal) and $z$ (vertical)

Table 6

Two-fluid simulation cases for the bed with high-amplitude vibration (configuration2).

\begin{tabular}{llll}
\hline Cases & $\begin{array}{l}\text { Mesh nodes (nodes in } \\
\text { horizontal direction) }\end{array}$ & $\begin{array}{l}\text { Vibration } \\
\text { frequency } f(\mathrm{~Hz})\end{array}$ & $\begin{array}{l}\text { Vibration } \\
\text { amplitude } A(\mathrm{~mm})\end{array}$ \\
\hline HA-1A & $2511(30)$ & No vibration & - \\
HA-1B & $9211(60)$ & & \\
HA-1C & $36,421(120)$ & & \\
HA-2A & $2511(30)$ & 15 & 15 \\
HA-2B & $9211(60)$ & 15 & 15 \\
HA-2C & $36,421(120)$ & 15 & 15 \\
HA-3 & $9211(60)$ & 15 & 5 \\
HA-4 & $9211(60)$ & 15 & 10 \\
HA-5 & $9211(60)$ & 20 & 5 \\
HA-6 & $9211(60)$ & 30 & 5 \\
\hline
\end{tabular}

direction in the region occupied by the bed particles. In cases SB $1 \mathrm{~B}$ and SB $2 \mathrm{~B}$ the mesh is refined by halving the cells size in $x$ and $z$ directions so that $\Delta s=0.892 \mathrm{~mm}$. Finally, after refining again the mesh in the two spatial directions, the cell size was reduced to $\Delta s=0.446 \mathrm{~mm}$ for cases SB 1C and SB 2C. Analogously, for the high amplitude vibrated fluidized bed, the cases HA $1 \mathrm{~A}$ and $\mathrm{HA}$ $2 \mathrm{~A}$ in Table 6 have a computational mesh composed of square cells whose size is $\Delta s=10 \mathrm{~mm}$ in the region containing particles. Refin ing in horizontal and vertical directions, the cell size is $\Delta s=5 \mathrm{~mm}$ for the cases $\mathrm{HA} 1 \mathrm{~B}$ and $\mathrm{HA} 2 \mathrm{~B}$, and $\Delta s=2.5 \mathrm{~mm}$ for the cases $\mathrm{HA}$ $1 \mathrm{C}$ and $\mathrm{HA} 2 \mathrm{C}$.

Fig. 4 depicts the mean and standard deviation of the gas pres sure at the distributor. To enhance visualization, both variables have been normalized dividing by the value obtained from the meshes of smallest cell size (i.e. meshes for SB $1 C$ and SB $2 \mathrm{C}$ in Ta ble 5 and for HA $1 \mathrm{C}$ and HA 2C in Table 6). The gas pressure was calculated performing the arithmetic mean of the local values of the gas static pressure at the distributor. Pressure is indicative of the bed dynamics and is affected by the interaction between the emulsion phase and the bubbles located anywhere in the bed. On view of Fig. 4a, it is clear that passing from the meshes SB $1 \mathrm{~B}$ and SB $2 B$ to the meshes SB $1 C$ and SB $2 C$ produces little differ ence in the results at the expense of increasing the computational cost, which was roughly proportional to the number of nodes in the mesh. Therefore, the computational mesh for the cases SB 1B and $\mathrm{SB} 2 \mathrm{~B}$ was selected to carry out the remaining simulations for the slim bed (i.e. cases SB 3 to SB 7 in 5). For similar reasons, see Fig. 4b, the mesh used in cases HA 1B and HA 2B was the one chosen to simulate the rest of cases in Table 6 for the high amplitude vibrated bed.

\subsection{Comparison with DEM results}

In this section the two fluid model methodology described in Section 3 is employed to simulate a slim bed of small scale, i.e. con figuration 1 in Table 4, previously characterized in the literature by Lagrangian Eulerian (DEM) simulations [23,24].

\subsubsection{Time evolution of particle volume fraction and pressure drop}

The evolution of the simulated particle volume fraction and gas pressure drop along time is first presented to elucidate whether the general dynamics of small vibrating beds can be satisfactorily predicted with two fluid models. The results presented here have been constructed by transversally averaging the simulated volume fraction and pressure along a horizontal line, covering the whole bed width, at a given distance from the distributor, $z$. Apart from the distributor, $z=0$, three different distances have been selected to perform the horizontal averaging, which are $z_{1}, z_{2}$ and $z_{3}$ in Fig. 2 b. From here on this averaging operation will be denoted as $x$ average.

Fig. 5a shows at different positions from the distributor the time variation of the $x$ average particle volume fraction taken from the simulation without vibration (case SB $1 \mathrm{~B}$ in Table 5). The same simulated variables are depicted in Fig. $5 \mathrm{~b}$ for the fluidized bed with vertical vibration at frequency $f_{z}=15 \mathrm{~Hz}$ with amplitude $A=1.5 \mathrm{~mm}$ (case SB 2B). In the case without vibration, Fig. 5a, near the bottom of the bed $(z=1.56 \mathrm{~mm})$, there is no appreciable change of particle volume fraction with time. The changes in vol ume fraction values, at higher positions in the bed, are due to the presence of voids (i.e. bubbles). In the case of the vibrated bed, Fig. 5b, a similar result to the one obtained with a DEM simulation strategy by Tatemoto et al. [23] is found: void fraction oscillations (1 $\left.\alpha_{p}\right)$ appear close to the distributor $(\mathrm{z}=1.563 \mathrm{~mm}$ ) at the same frequency of the imposed vibration, i.e. $15 \mathrm{~Hz}$. Moreover, at this lower position, the $x$ averaged particle volume fraction oscillates along time around a mean volume fraction value that is lower than 

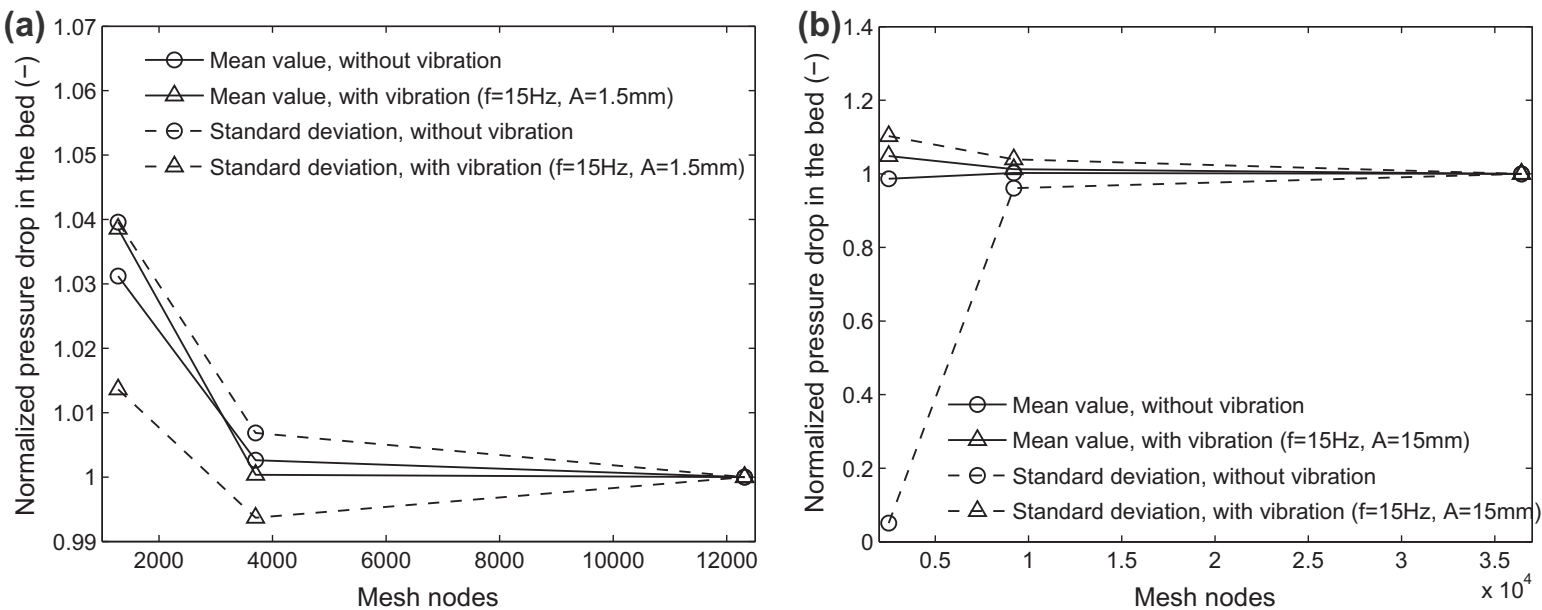

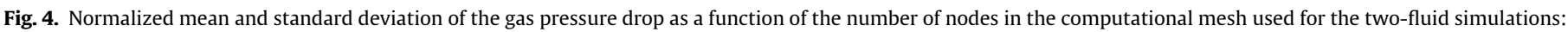
(a) slim bed; (b) high-amplitude vibrating bed.
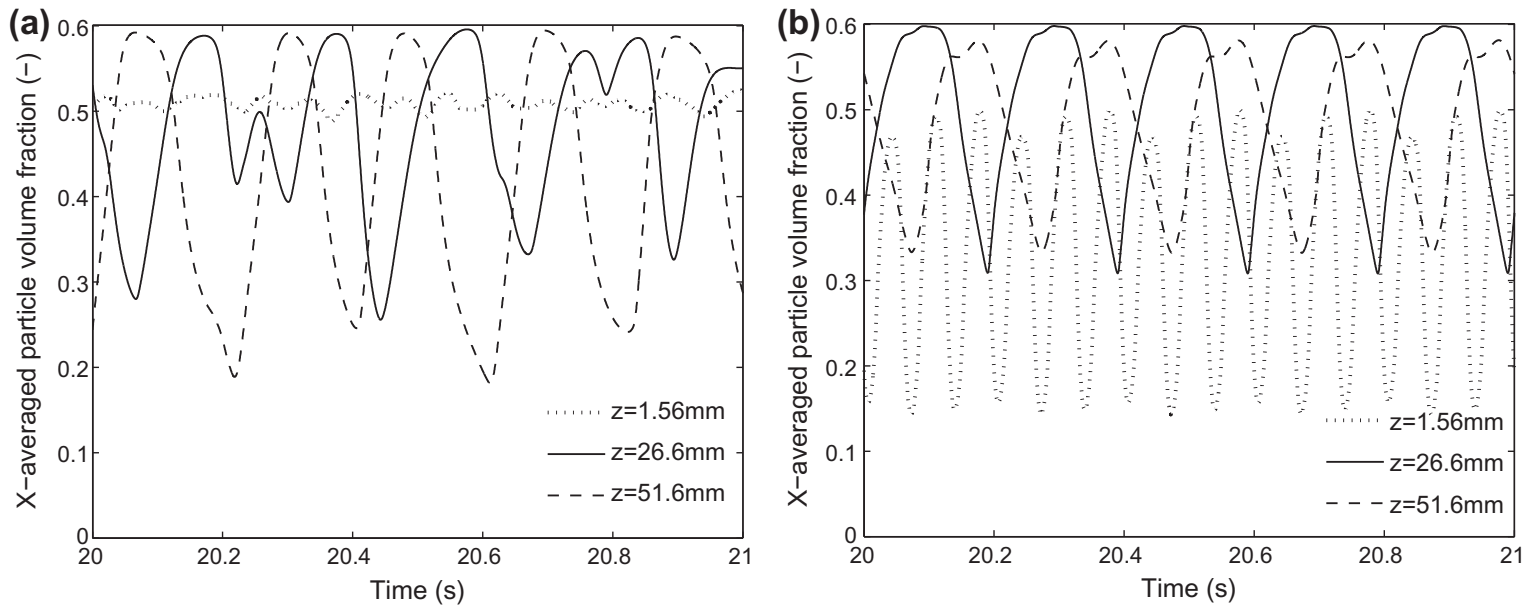

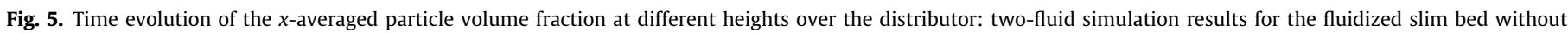
vibration (a), and with vibration at $f=15 \mathrm{~Hz}$ and $A=1.5 \mathrm{~mm}$ (b).

for the case without vibration. This behavior was also reported in Tatemoto et al. [23] and Xiang et al. [24], who showed that a void gap appears close to the distributor at high frequencies that lowers the mean volume fraction. At higher positions in the bed, $z=26.6 \mathrm{~mm}$ and $51.6 \mathrm{~mm}$ in Fig. 5b, the time evolution of the $x$ averaged volume fraction does not closely follow the imposed vibration and have a pattern that resembles more the solid volume fraction of the bed without vibration, $z=26.6 \mathrm{~mm}$ and $51.6 \mathrm{~mm}$ in Fig. 5a. Thus, the presence of bubbles is probably the dominant phenomenon affecting the particle volume fraction variation at these higher positions in the bed.

Simulated pressure signals are presented in Fig. 6 for the two fluid model cases without vibration (SB 1B) with vibration at $15 \mathrm{~Hz}$ and displacement $1.5 \mathrm{~mm}$ (SB 2B). The pressure signal was obtained by $x$ averaging the air relative pressure close to the dis tributor in the simulated bed. Here, the air relative pressure is the air pressure minus the air pressure at the top of the freeboard. Thus, the pressure signal can be interpreted as the pressure drop that suffers the air when passing through the whole bed. As for the particle volume fraction signals, a higher number of pressure oscillations are found in the case with vibration compared to the non vibrated case. This two fluid simulation outcome is in har mony with the results obtained with DEM simulations by Xiang et al. [24]. The vertical vibration of the bed creates an oscillatory bulk motion of the bed, which directly affects the pressure at the

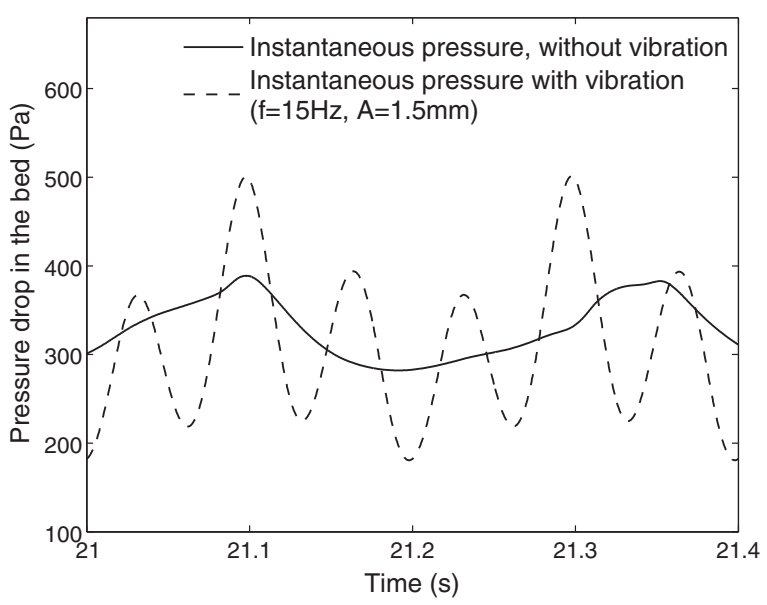

Fig. 6. Instantaneous and mean gas pressure drop in the fluidized slim bed. The results were obtained from the two-fluid simulations without vibration and with vibration at $f=15 \mathrm{~Hz}$ and $A=1.5 \mathrm{~mm}$. 

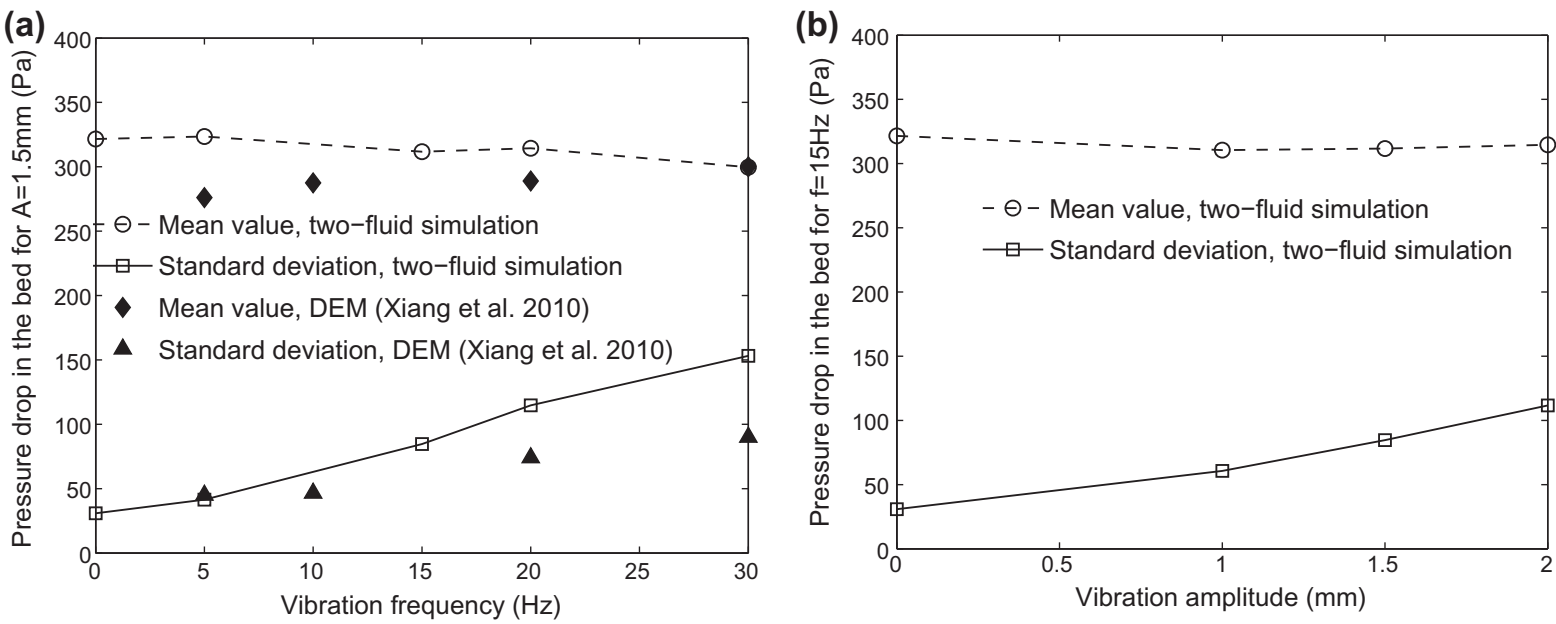

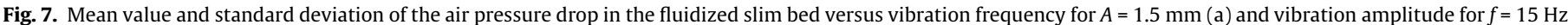
(b)

(a)

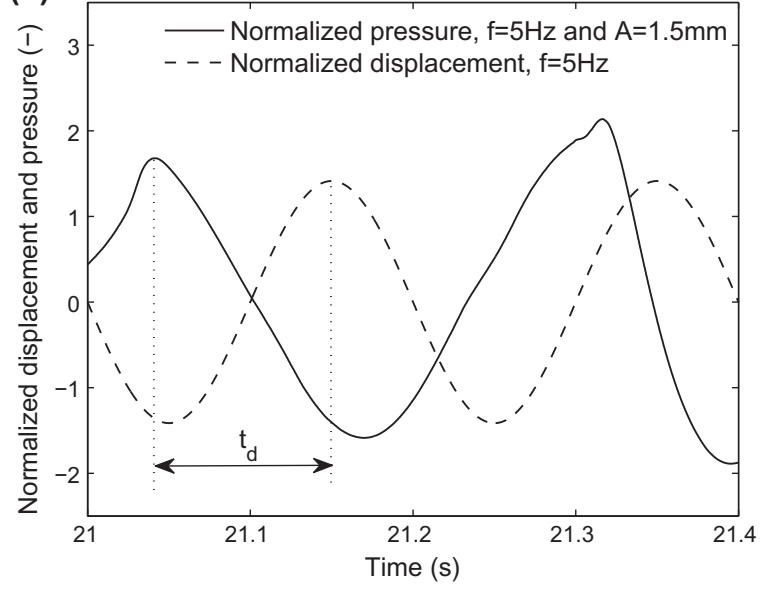

(b)

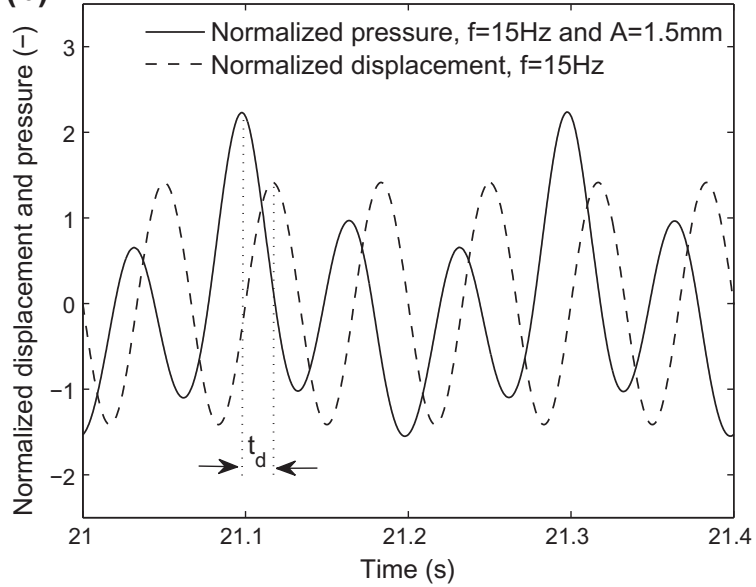

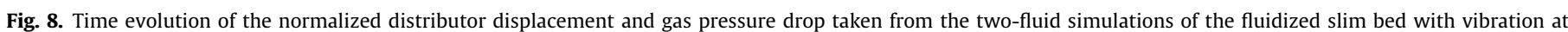
$f=5 \mathrm{~Hz}(\mathrm{a})$ and $f=15 \mathrm{~Hz}$ (b). In both cases $A=1.5 \mathrm{~mm}$.

distributor. According to Fig. 6, the main frequency of the pressure oscillation in the simulations is similar to the vibration frequency imposed to the bed $(15 \mathrm{~Hz})$. On the contrary, as shown in Fig. 6, the frequency of the simulated pressure drop for the bed without vibration coincides with the natural frequency of the bed esti mated with $f_{n} \approx\left(g / h_{0}\right)^{1 / 2} / \pi=4.3 \mathrm{~Hz}$ [36]. This natural frequency is still present in the vibrated fluidized bed since the amplitude of the $15 \mathrm{~Hz}$ pressure drop oscillations in Fig. 6 are modulated with a frequency equal to $f_{n}$.

\subsubsection{Effect of vibration frequency and amplitude}

The values obtained for the simulated mean and the standard deviation of the pressure drop, at different vertical vibration fre quencies, are represented in Fig. 7a. For that purpose, cases SB $1 \mathrm{~B}, \mathrm{SB} 2 \mathrm{~B}, \mathrm{SB} 3, \mathrm{SB} 4$ and SB 5 in Table 5 have been used in the present analysis. Increasing the vibration frequency entails higher values of the standard deviation of pressure fluctuations, while their mean is almost constant, that is, independent of vibration. This trend is in good agreement with the results obtained in the DEM simulation by Xiang et al. [24]. The effect of the vibration amplitude is shown in Fig. 7b. As with the frequency, the mean pressure remains almost constant whereas the standard deviation of the pressure drop increases with the vertical vibration amplitude.

In Fig. 8, the normalized pressure fluctuations and distributor displacement over time are shown for two different vibration fre quencies. Normalization was done by subtraction of the mean va lue and division by the standard deviation of each signal. As commented previously, the bed vibration frequency is recovered in the simulated pressure fluctuation curve, but with a certain de lay time. Following Xiang et al. [24], the delay time is defined as the difference between the time at the maximum value of pressure and the time of maximum displacement in the distributor (see Fig. 8).

The delay time is characterized in Fig. 9. According to the two fluid simulation results, the delay time is highly dependent on the vibration frequency, as shown in Fig. 9a. A change in the vibration frequency modifies the vibration time period $(1 / f)$ which alters the available time for the particles to reproduce the bed vessel vibra tion. In contrast, the vibration time period remains constant in Fig. $9 \mathrm{~b}$ and, as the two fluid simulations reveal, the effect of the vibration amplitude on the delay time is not as sharp. This ten dency is in fine agreement with the DEM results reported in Xiang et al. [24]. Though quantitative differences appear between the 

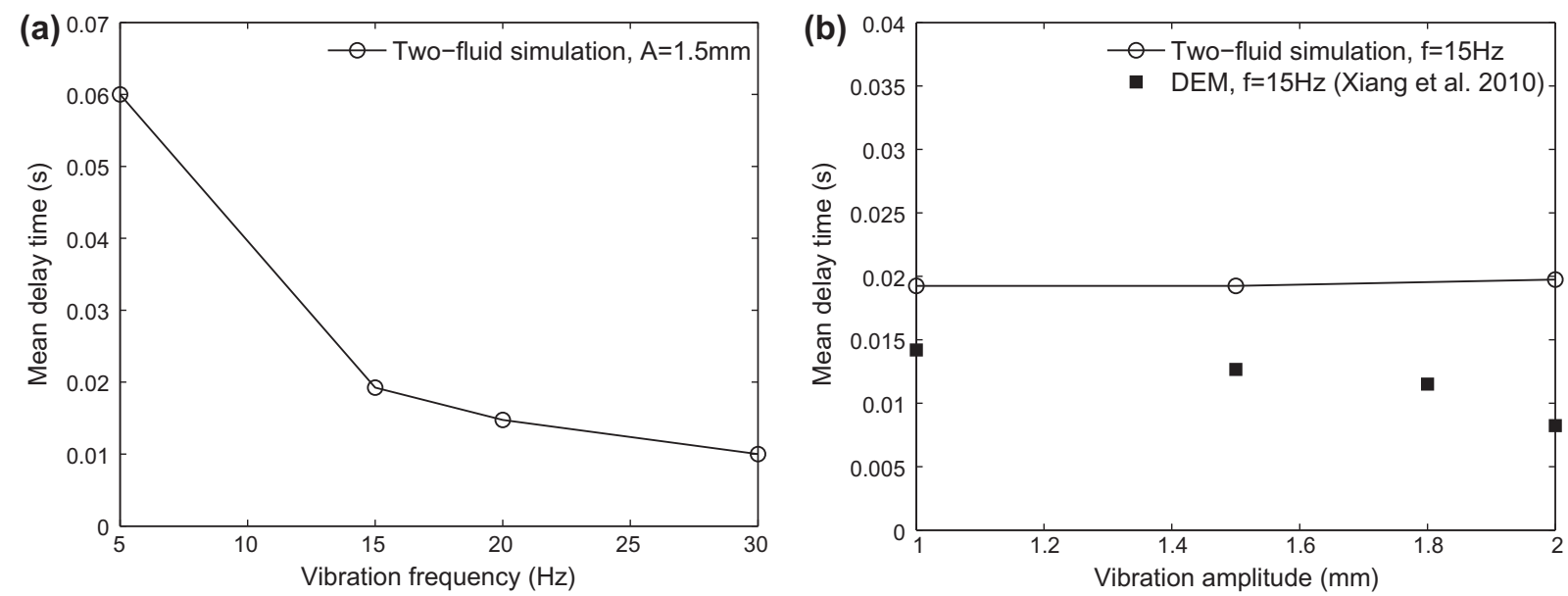

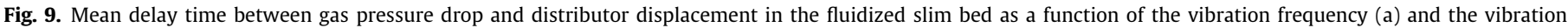
amplitude (b).
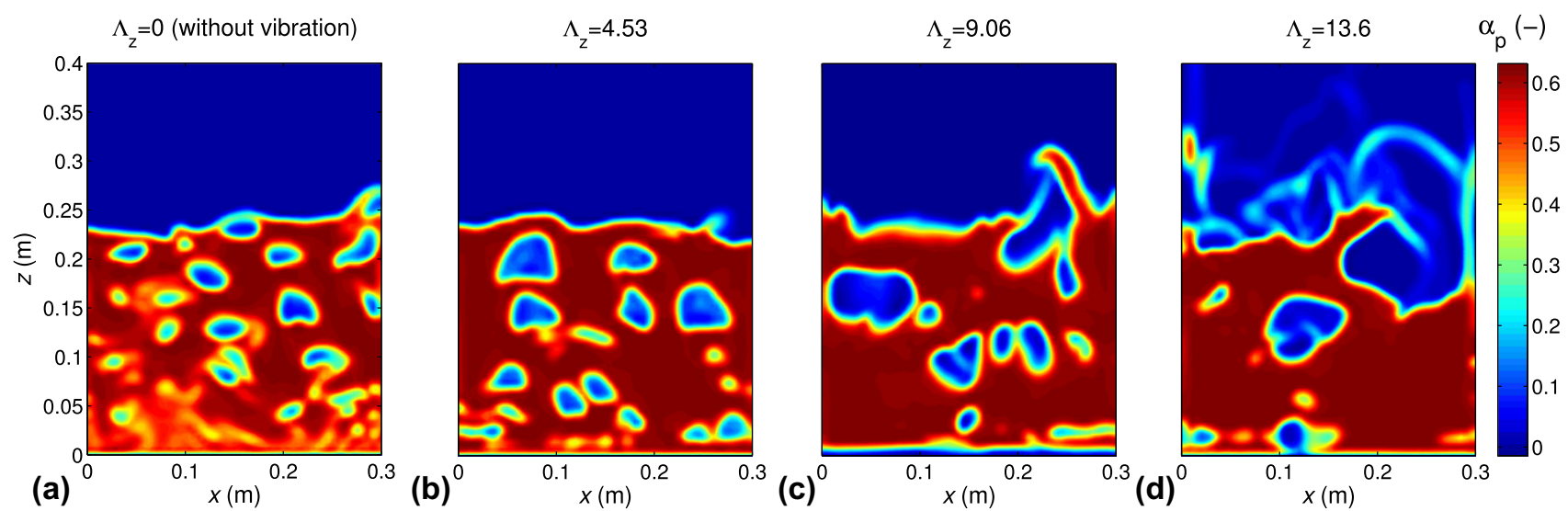

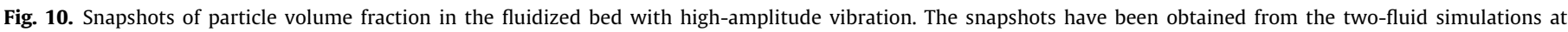
$f=15 \mathrm{~Hz}$ and different vibration strengths: (a) $\Lambda_{z}=0(A=0 \mathrm{~mm}) ;(\mathrm{b}) \Lambda_{z}=4.53(A=5 \mathrm{~mm}) ;(\mathrm{c}) \Lambda_{z}=9.06(A=10 \mathrm{~mm}) ;(\mathrm{d}) \Lambda_{z}=13.6(A=15 \mathrm{~mm})$.

two fluid simulation and DEM results in Fig. 9b, the obtained val ues for the delay time are of the same order of magnitude, which is a remarkable finding considering that the fluid like behavior of the particle phase (an assumption implicit in two fluid models) may not be fully reached if the number of particles is small as in the case of this slim bed configuration.

\subsection{Comparison with experiments}

In the previous section it has been seen that the two fluid model methodology presented here can provide comparable results to the Lagrangian Eulerian simulation of a fluidized bed of small scale under reduced vibration amplitudes. In order to extend this assessment of capabilities of two fluid models in simulating vibrated beds, the next results are focused on a larger bed with high amplitude vibration (configuration 2 in Table 6). The results from the two fluid model simulation are compared with the experimental findings shown in Zhou et al. [19] and Mawatari et al. [20].

\subsubsection{Effect of vibration on the bubble behavior}

Fig. 10 shows four snapshots of the instantaneous solids volume fraction distribution obtained with two fluid model simulations at different vibration intensities characterized by the vibration strength parameter [1]

$$
\Lambda_{z} \frac{4 \pi^{2} A f_{z}^{2}}{\left|g_{z}\right|}
$$

The values of $\Lambda_{z}$ in Fig. 10 correspond to a fixed vibration frequency of $15 \mathrm{~Hz}$ while the vibration amplitude takes the values of $0,5,10$ and $15 \mathrm{~mm}$ (cases HA 1B, HA 2B, HA 3 and HA 4 in Table 6). The presence of bubbles in all cases shown in Fig. 10 demonstrates the bubbling condition of the bed without and with vibration.

Apparently, an increase of the vibration amplitude promotes the presence of larger bubbles in the bed. This effect is in good agreement with the experimental photographs obtained in $[19,20]$. The rationale of this effect is that an increase of the vibra tion amplitude creates higher acceleration forces suffered by the bed during the sinusoidal displacement of the vessel. In other words, the vertical vibration strength is more intense with larger vibration amplitudes $A$. As observed by Mawatari et al. [20], the increasing of the vibration strength reduces the minimum fluidiza tion velocity and, as a consequence, increases excess gas velocities, so the expected visible flow in form of bubbles grows. Besides, it has been reported that increasing of the bed agitation amplitude, produced when increasing $A$, can favor the coalescence of bubbles [19], which is a way of increasing the size of the bubbles.

The effect of vibration frequency on the local mean diameter of bubbles is illustrated in Fig. 11a for a vibration amplitude equal to $5 \mathrm{~mm}$ (cases HA 1B and HA 4 to 6 in Table 6). In the figure, simu 

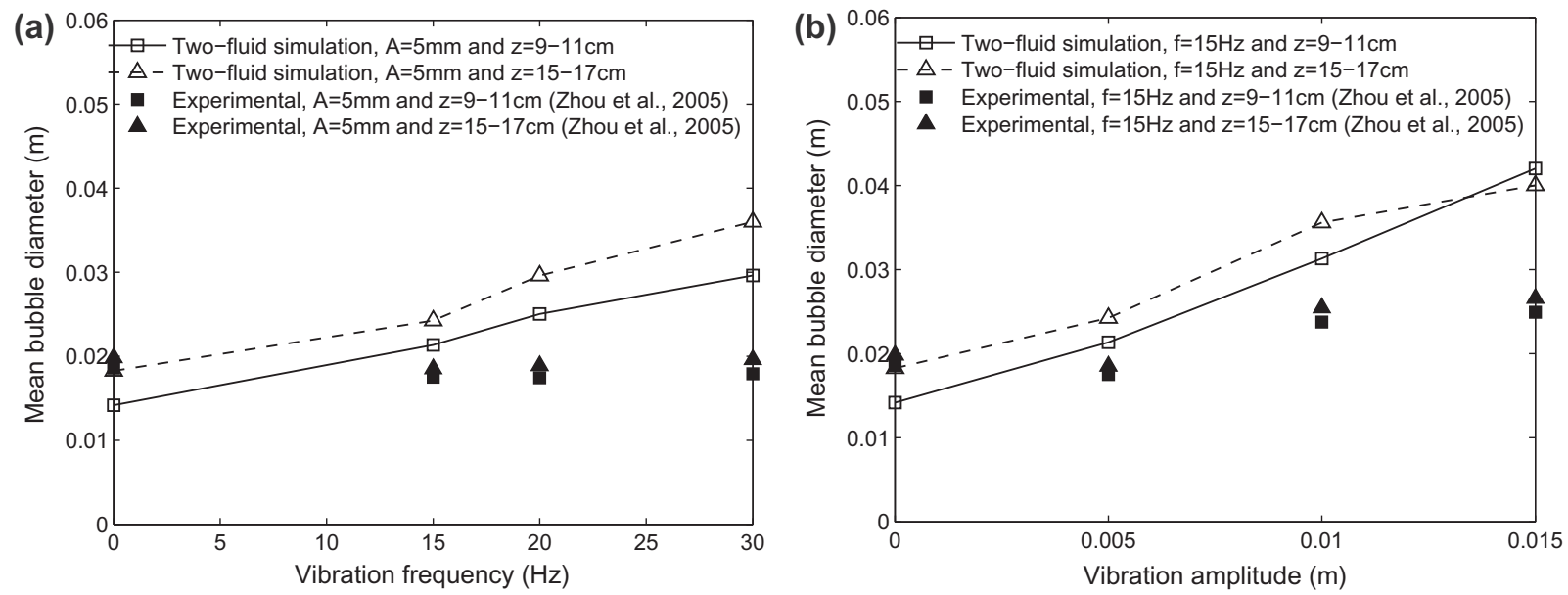

Fig. 11. Mean diameter of bubbles in the fluidized bed with high-amplitude vibration as a function of the vibration frequency for $A=5 \mathrm{~mm}$ (a), and the vibration amplitude for $f=15 \mathrm{~Hz}(\mathrm{~b})$.
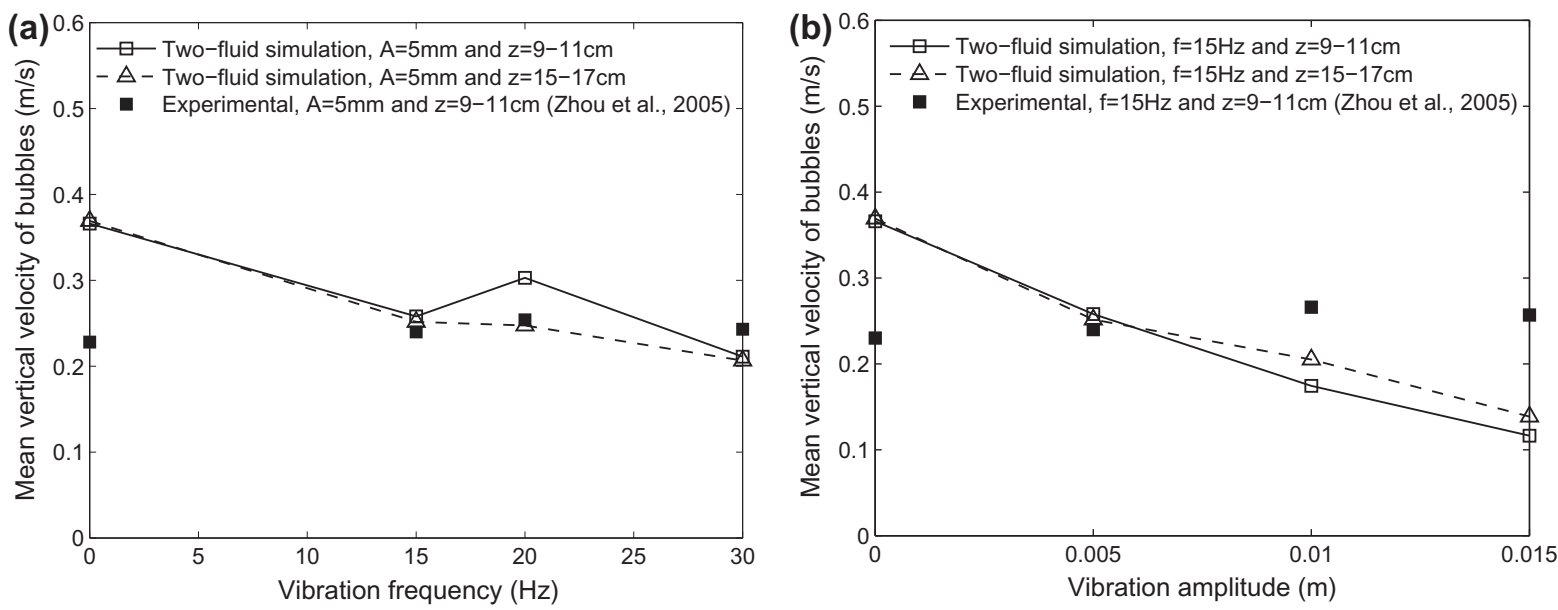

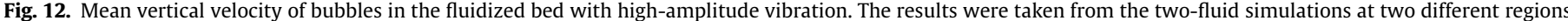
over the distributor as a function of the vibration frequency for $A=5 \mathrm{~mm}$ (a) and the vibration amplitude for $f=15 \mathrm{~Hz}$ (b).

lation results at two different vertical distances, $z$, from the distrib utor are compared with the ones experimentally obtained by Zhou et al. [19].

Bubbles are detected in the simulation from snapshots of solids volume fraction exported every $5 \times 10^{3} \mathrm{~s}$ of computed time. A threshold value of particle volume fraction equal to $\alpha_{p, t h}=0.3$ was used to detect the bubble contour. Information concerning the selection of $\alpha_{p, t h}$ and the calculation of the bubble diameter and velocity from the bubble contours can be found elsewhere [28]. As in Zhou et al. [19], bubbles are captured when their cen troids pass through any of the two sampling regions marked in Fig. 3b. In each sampling region, the mean diameter is calculated performing the arithmetic mean of the diameters of all the cap tured bubbles. These two sampling regions are relatively thin $(2 \mathrm{~cm})$ and span all the bed width; one region ranges from $z=9 \mathrm{~cm}$ to $11 \mathrm{~cm}$, and the other region from $z=15 \mathrm{~cm}$ to $17 \mathrm{~cm}$. As Fig. 11a indicates, the mean bubble diameter obtained with the two fluid simulations and the experiments are reasonably sim ilar. The simulations show that the bubble size increases with the vibration frequency, though this increment is not evident in the case of the experimental data. Regarding the vibration amplitude, Fig. 11b shows that the mean bubble diameter obtained from the two fluid simulations (cases HA 1B, HA 2B, HA 3 and HA 4 in Ta ble 6 ) increases with the vibration amplitude. As Fig. 11b demon strates, this result is in harmony with the experimental data reported in [19]. Also in good agreement with the experimental evidence is the fact that the mean bubble diameter seems to be more sensitive to the vibration amplitude than to the vibration fre quency (i.e. doubling the vibration amplitude alters the mean bub ble diameter more than doubling the vibration frequency). Besides, both the simulated and experimentally acquired bubbles have slightly larger diameters when increasing the distance of the sam pling region to the distributor, $z$.

The mean vertical velocity of bubbles obtained from the two fluid model simulations as a function of the vibration frequency and amplitude is presented in Fig. 12. The results have been taken from the same simulation cases utilized in Fig. 11. In addition, experimental bubble velocity data from [19] have been included for comparison purposes. In Fig. 12 the mean velocity depicted is relative to the bed distributor movement. It was found that expressing the results in an absolute system of reference did not change significantly the mean velocity because the instantaneous differences between the relative and absolute velocity are compen sated after calculating the mean over the time.

Both the two fluid simulation and the experiments yield mean bubble velocities of the same order or magnitude. In some cases (see vibration with $A=5 \mathrm{~mm}$ and $f=15 \mathrm{~Hz}$ ) the value of the mean bubble velocity provided by the two fluid simulation is virtually 

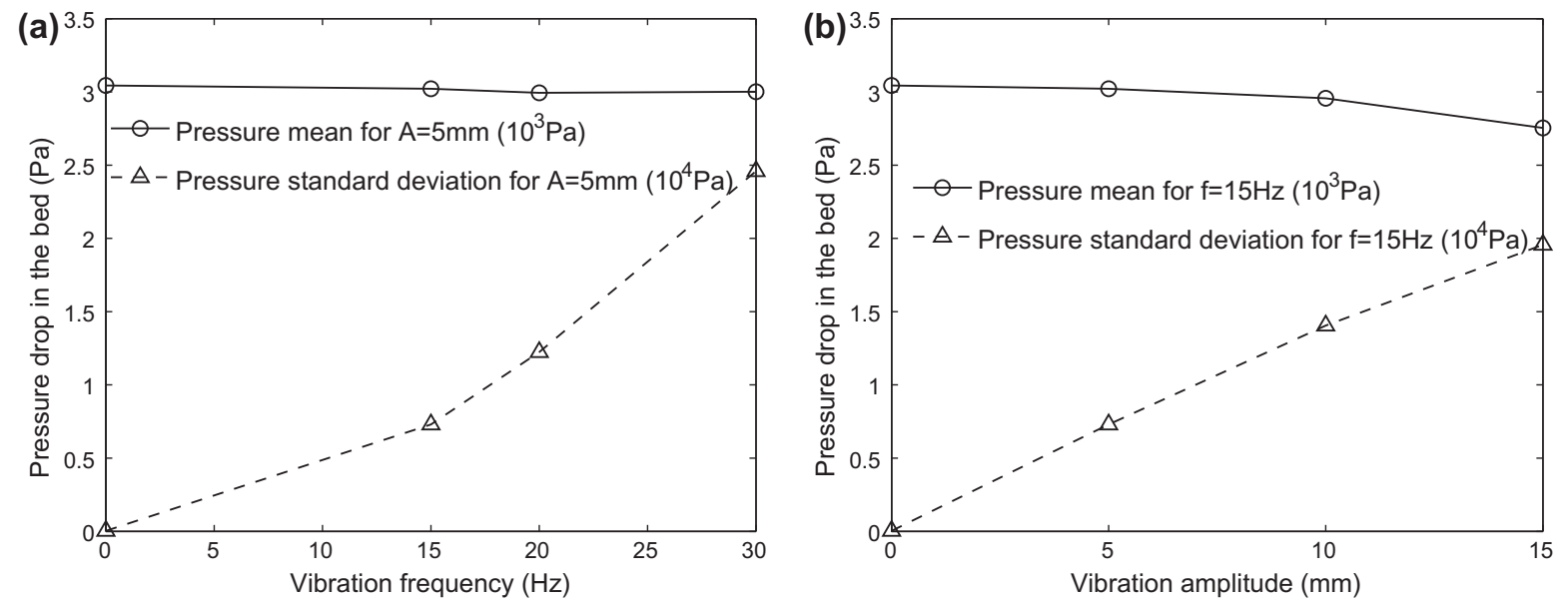

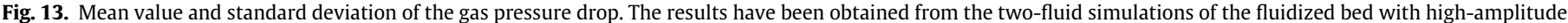
vibration as a function of the vibration frequency for $A=5 \mathrm{~mm}$ (a), and the vibration amplitude for $f=15 \mathrm{~Hz}$ (b).

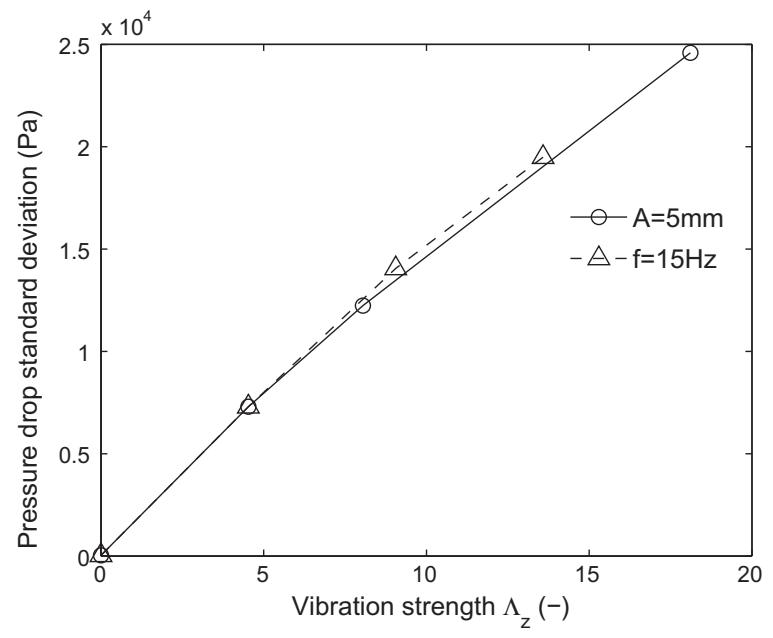

Fig. 14. Standard deviation of the gas pressure drop versus the vibration strength The results have been taken from the two-fluid simulations shown in Fig. 13.

equal to the experimental data. According to Fig. 12a, changes in the vibration frequency do not produce a clear tendency on the change of the mean vertical velocity estimated with the two fluid simulation results at $z=911 \mathrm{~cm}$, which is in agreement with the experimental results. Unexpectedly, the major discrepancies be tween the simulation and the experimental data appear for the bed without vibration $(f=0 \mathrm{~Hz})$. The effect of the vibration ampli tude on the mean vertical velocity of bubbles is illustrated in Fig. 12b. Again, the bubble velocities obtained from the simulation and from the experiments by Zhou et al. [19] have the same order of magnitude. Bubbles in the experiments have a mean velocity that slightly increases and then decreases with the vibration amplitude, though this dependency is weak. In contrast, the ten dency revealed by the two fluid simulation is that the bubble velocity monotonely decreases with the vibration amplitude. How ever, it is worth mentioning that the estimation of the bubble velocity in simulations and experiments is an operation sensitive to the way the displacement of the bubble centroid is calculated. Changes in shape of an irregular bubble may produce an apparent displacement that is difficult to separate from what would be ex pected to be the ascending velocity of the bulk volume of the bub ble. In fact, bubbles in VFB can be larger and with more irregular boundaries than in conventional FB. In experimental vibrating beds the estimation of the bubble velocity has the additional burden, not encountered in the simulation, of the matching between the camera and the bed vessel vibration, which can penalize the reso lution of the bubble boundaries in the particle images. These fac tors could contribute to the differences encountered between the two fluid simulations and the experimental results concerning the mean velocity of bubbles. Nevertheless the ultimate causes of the differences between the present two fluid simulations and the experiments by Zhou et al. [19] are unknown and further experimental data and computational studies are required to give an answer.

\subsubsection{Effect of vibration on the pressure drop}

Accordingly to the previous results, since the main dynamics of the bed is promoted by the presence of bubbles, and they become larger in size while varying the vibration strength, an analogous re sult to that obtained for the slim bed (configuration 1 in Section 4.2.2) is expected for the high amplitude vibrated bed (configura tion 2). In particular, as the two fluid simulations presented in Fig. 13a indicate, the time averaged value of the pressure drop in the bed remains practically unchanged with the vibration fre quency whereas the standard deviation of the pressure drop in creases more than proportionally, i.e. in an approximately quadratic way. This trend is not so clear in the slim bed (configu ration 1) where particles occupy a narrow volume affected by the presence of walls. When the vibration amplitude is augmented, Fig. 13b, the standard deviation also increases. However the stan dard deviation of the pressure drop in the bed grows linearly. The different growth rate of the standard deviation with frequency and amplitude can be explained resorting to the vibration strength, which is naturally incorporated through the equivalent mass force used in the proposed methodology for the two fluid simulation. In particular, after introducing Eq. (13) into Eq. (12) for the case of downward gravity acceleration, i.e. $g_{z}=9.81 \mathrm{~m}^{2} / \mathrm{s}$, it is easy to see that:

$\mathbf{f}_{m, e q} \quad\left\{0,0, g_{z}\left[1 \quad \Lambda_{z} \sin \left(2 \pi f_{z} t\right)\right]\right\}$

According to Eq. (14), an increase of the vibration strength aug ments the fluctuating intensity of the equivalent mass force $\mathbf{f}_{m, e q}$. Therefore, given that the vibration strength is proportional to the amplitude and the square of the frequency, Eq. (13), it seems evi dent that the vibration strength is the parameter that actually con trols the magnitude of the oscillations observed for the gas pressure drop in a vibrated fluidized bed. This is confirmed in Fig. 14, in which all the results for the pressure standard deviation of Fig. 13a and tend to collapse on the same curve as a function of the vertical vibration strength $\Lambda_{z}$. 


\section{Summary and conclusions}

A novel methodology to simulate vibrating beds using Eulerian Eulerian two fluid models was presented in this work. The method is based on expressing the governing equations in a moving system of reference for which vibration is incorporated through equivalent body forces. Thus, the method is relatively simple to implement. It eliminates the need for moving boundaries to cause vibration and the computational cost is similar than that for two fluid models applied to conventional non vibrating beds. According to the ob tained results, the dynamics of a slim vibrating fluidized bed sim ulated with the proposed two fluid model strategy was in reasonable agreement with the results of previous Lagrangian Eulerian simulations (DEM) present in the literature. This agree ment is remarkable considering that the small number of particles contained in the studied slim bed may be insufficient to fully reach the fluid like behavior of the particle phase that is assumed in two fluid models. Comparison with reported experimental studies of a vibrating quasi two dimensional bed seems to indicate that two fluid models are capable of predicting realistically the bubble behavior in vibrated fluidized beds even if the vibration is of large amplitude. Nevertheless, some discrepancies between the simulation results and the experimental measurements concerning the mean vertical velocity of bubbles would require further confir mation with additional experimental data.

The potential applications of the two fluid methodology pre sented here are significant. As two fluid models are able to deal with beds having larger amounts of particles than Lagrangian (DEM) models, the two fluid model methodology here described opens the way of dramatically increasing the scale of the vibrated fluidized beds currently simulated. Furthermore, nanofluidization processes that are mechanically assisted by vibration to reduce agglomeration can also benefit from the use of the two fluid mod els. Due to the extremely reduced size of nano powders, the re quired amount of these ultrafine particles is very large even in beds of small scale. Alternative simulations strategies, such as the proposed two fluid methodology (with appropriate closure models), can help advance the understanding and modeling of nanofluidization.

\section{Acknowledgements}

The present work has been funded by the Spanish Ministerio de Ciencia e Innovación through the Project DPI2009 10518. The Authors gratefully appreciate this support.

\section{References}

[1] C. Strumillo, Z. Pakowski, Drying of granular products in vibrofluidized beds, in: A.S. Mujumdar (Ed.), Drying'80: Developments in Drying, Hemisphere Publishing Corporation, Montreal, 1980, pp. 211-226.

[2] D. Reay, C.G.J. Baker, Drying, in: J.F. Davidson, R. Clift, D. Harrison (Eds.), Fluidization, second ed., Academic Press, London, 1985, pp. 529-562.

[3] S. Mori, A. Yamamoto, S. Iwata, T. Haruta, I. Yamada, E. Mizutani, Vibrofluidization of group $C$ particles and its industrial applications, AIChE Symp. Ser. 86 (1990) 88-94.

[4] N.J.M. Kuipers, E.J. Stamhuis, A.A.C.M. Beenackers, Fluidization of potato starch in a stirred vibrating fluidized bed, Chem. Eng. Sci. 51 (1996) 2727-2732.

[5] H. Kage, M. Oba, H. Ishmatsu, H. Ogura, Y. Matsuno, The effects of frequency and amplitude on the powder coating of fluidizing particles in vibro-fluidized bed, Adv. Powder Technol. 10 (1999) 77-87.

[6] W. Zhang, A review of techniques for the process intensification of fluidized bed reactors, Chin. J. Chem. Eng. 17 (2009) 688-702.
[7] C.H. Nam, R. Pfeffer, R.N. Dave, S. Sundaresan, Aerated vibrofluidization of silica nanoparticles, AIChE J. 50 (2004) 1776-1785.

[8] J.M. Valverde, A. Castellanos, Fluidization of nanoparticles: a simple equation for estimating the size of agglomerates, Chem. Eng. J. 140 (2008) 296-304.

[9] K. Noda, Y. Mawatari, S. Uchida, Flow patterns of fine particles in a vibrated fluidized bed under atmospheric or reduce pressure, Powder Technol. 99 (1998) 11-14.

[10] A.S. Mujumbar, Handbook of Industrial Drying, Marcel Dekker, New York, 1987.

[11] E. Bratu, G.I. Jinescu, Effect of vertical vibrations on the pressure drop in a fluidized layer, Br. Chem. Eng. 16 (1971) 691-695.

[12] T.-J. Wang, Y. Jin, A. Tsutsumi, Z. Wang, Z. Cui, Energy transfer mechanism in a vibrating fluidized bed, Chem. Eng. J. 78 (2000) 115-123.

[13] J. Werther, Bubbles in gas fluidised beds - part I, Trans. Inst. Chem. Eng. 52 (1974) 149-159.

[14] D.J. Patil, M. van Sint Annaland, J.A.M. Kuipers, Critical comparison of hydrodynamics models for gas-solid fluidized beds - part II: freely bubbling gas-solid fluidized beds, Chem. Eng. Sci. 60 (2004) 73-84.

[15] A. Busciglio, V. Giuseppa, G. Micale, L. Rizzuti, Analysis of the bubbling behavior of 2D gas solid fluidized beds. Part I. Digital image analysis technique, Chem. Eng. J. 140 (2008) 398-413.

[16] C. Sobrino, J.A. Almendros-Ibáñez, D. Santana, C. Vázquez, M. de Vega, Maximum entropy estimation of the bubble size distribution in fluidized beds, Chem. Eng. Sci. 64 (2009) 2307-2319.

[17] A. Acosta-Iborra, C. Sobrino, F. Hernández-Jiménez, M. de Vega, Experimental and computational study on the bubble behavior in a 3-D fluidized bed, Chem. Eng. Sci. 66 (2011) 3499-3512.

[18] T. Zhou, H. Kage, S. Funaoka, H. Ogura, Y. Matsuno, Fluidization behavior of glass beads under different vibration modules, Adv. Powder Technol. 12 (2001) 559-575.

[19] T. Zhou, H. Kage, H. Li, Bubble characteristics in a two-dimensional vertically vibro-fluidized bed, China Particuol. 3 (2005) 224-228.

[20] Y. Mawatari, K. Tagawa, Y. Tatemoto, K. Noda, J. Chem. Eng. Jpn. 38 (2005) $18-23$.

[21] P.A. Cundall, O.D.L. Strack, Discrete numerical-model for granular assemblies, Geotechnique 29 (1979) 47-65.

[22] B.G.M. van Wachem, A.E. Almstedt, Methods for multiphase computational fluid dynamics, Chem. Eng. J. 96 (2003) 81-98.

[23] Y. Tatemoto, Y. Mawatari, T. Yasukawa, K. Noda, Numerical simulation of particle motion in vibrated fluidized bed, Chem. Eng. Sci. 59 (2004) 437-447.

[24] L. Xiang, W. Shuyan, L. Huilin, L. Goudong, C. Juhui, L. Yikun, Numerica simulation of particle motion in vibrated fluidized beds, Powder Technol. 197 (2010) 25-35.

[25] S. Limtrakul, W. Rotjanavijit, T. Vatanatham, Lagrangian modeling and simulation of effect of vibration on cohesive particle movement in a fluidized bed, Chem. Eng. Sci. 62 (2007) 232-245.

[26] D. Gidaspow, Multiphase Flow and Fluidization, Academic Press, Boston, 1994.

[27] A. Busciglio, V. Giuseppa, G. Micale, L. Rizzuti, Analysis of the bubbling behavior of 2D gas solid fluidized beds. Part II. Comparison between experiments and numerical simulations via digital image analysis technique, Chem. Eng. J. 148 (2008) 145-163.

[28] F. Hernández-Jiménez, S. Sánchez-Delgado, A. Gómez-García, A. Acosta-Iborra Comparison between two-fluid model simulations and particle image analysis \& velocimetry (PIV) results for a two-dimensional gas-solid fluidized bed, Chem. Eng. Sci. 66 (2011) 3753-3772.

[29] F. Hernández-Jiménez, J.R. Third, A. Acosta-Iborra, C.R. Müller, Comparison of bubble eruption models with two-fluid simulations in a 2D gas-fluidized bed, Chem. Eng. J. 171 (2011) 328-339.

[30] D. Gidaspow, R. Bezburuah, J. Ding, Hydrodynamics of circulating fluidized beds, kinetic theory approach, in: Fluidization VII, Proceedings of the 7th Engineering Foundation Conference on Fluidization, 1992, pp. 75-82.

[31] C.K.K. Lun, S.B. Savage, D.J. Jeffrey, N. Chepurniy, Kinetic theories for granular flow: inelastic particles in Couette flow and slightly inelastic particles in a general flow field, J. Fluid Mech. 140 (1984) 223-256.

[32] D.G. Schaeffer, Instability in the evolution equations describing incompressible granular flow, J. Diff. Eqns. 66 (1987) 19-50.

[33] N. Menon, D.J. Durian, Particle motions in a gas-fluidized bed of sand, Phys. Rev. Lett. 79 (1997) 3407-3410.

[34] S. Sánchez-Delgado, C. Marugán-Cruz, A. Acosta-Iborra, D. Santana, Densephase velocity fluctuation in a 2-D fluidized bed, Powder Technol. 200 (2010) 37-45.

[35] P.C. Johnson, R. Jackson, Frictional-collisional constitutive relations for granular materials, with application to plane shearing, J. Fluid Mech. 176 (1987) 67-93.

[36] A.P. Baskakov, V.G. Tuponogov, N.F. Filippovsky, A study of pressure fluctuations in a bubbling fluidized bed, Powder Technol. 45 (1986) 113-117. 